\title{
The Herschel-PACS photometer calibration
}

\section{Point-source flux calibration for scan maps}

\author{
Zoltan Balog · Thomas Müller · Markus Nielbock · \\ Bruno Altieri · Ulrich Klaas · Joris Blommaert · \\ Hendrik Linz · Dieter Lutz · Attila Moór · Nicolas \\ Billot · Marc Sauvage · Koryo Okumura
}

Received: date / Accepted: date

\begin{abstract}
This paper provides an overview of the PACS photometer flux calibration concept, in particular for the principal observation mode, the scan map. The absolute flux calibration is tied to the photospheric models of five fiducial stellar standards ( $\alpha$ Boo, $\alpha$ Cet, $\alpha$ Tau, $\beta$ And, $\gamma$ Dra). The data processing steps to arrive at a consistent and homogeneous calibration are outlined. In the current state the relative photometric accuracy is $\sim 2 \%$ in all bands. Starting from the present calibration status, the characterization and correction for instrumental effects affecting the relative calibration accuracy is described and an outlook for the final achievable calibration numbers is given. After including all the correction for the instrumental effects, the relative photometric calibration accuracy (repeatability) will be as good as $0.5 \%$ in the blue and green band and $2 \%$ in the red band. This excellent calibration starts to reveal possible inconsistencies between the models of the K-type and the Mtype stellar calibrators. The absolute calibration accuracy is therefore mainly limited by the $5 \%$ uncertainty of the celestial standard models in all three bands. The PACS bolometer response was extremely stable over the entire Herschel mission and a single, time-independent
\end{abstract}

\section{Z. Balog}

Max-Planck-Institut für Astronomie, Königstuhl 17, D-69117 Heidelberg Germany

Tel.: +496221 528-354

E-mail: balog@mpia.de

T. Müller, D. Lutz

Max-Planck-Institut für Extraterrestrische Physik, Garching, Germany

M. Nielbock, U. Klaas, H. Linz

Max-Planck-Institut für Astronomie, Heidelberg, Germany

B. Altieri

ESAC, Madrid, Spain

J.A.D.L. Blommaert

Instituut voor Sterrenkunde, K.U.Leuven, Leuven, Belgium

A. Moór

Konkoly Observatory, Budapest, Hungary

N. Billot

IRAM, Granada, Spain

M. Sauvage, K. Okumura

CEA, Saclay, France 
response calibration file is sufficient for the processing and calibration of the science observations. The dedicated measurements of the internal calibration sources were needed only to characterize secondary effects. No aging effects of the bolometer or the filters have been found. Also, we found no signs of filter leaks. The PACS photometric system is very well characterized with a constant energy spectrum $v F_{v}=\lambda F_{\lambda}=$ const as a reference. Colour corrections for a wide range of sources SEDs are determined and tabulated.

Keywords Herschel Space Observatory · PACS · Far-infrared · Instrumentation · Calibration $\cdot$ Scan-map

\section{Introduction}

The Photodetector Array Camera and Spectrograph (PACS) [17] on board the Herschel Space Observatory [15] provides photometric imaging and integral field spectroscopy capabilities for the far-infrared wavelength regime. The PACS photometer imaging unit is a dual band imaging camera. It allows the simultaneous observation in two bands, either in the combination $70 \mu \mathrm{m}($ blue $)+160 \mu \mathrm{m}$ (red) or $100 \mu \mathrm{m}($ green $)+160 \mu \mathrm{m}$ (red), where 70 or $100 \mu \mathrm{m}$ bands are selected by a filter wheel. The camera contains two bolometer detector arrays with $64 \times 32$ pixels (blue array) and $32 \times 16$ pixels (red array), respectively, providing an instantaneous field-of-view of $3.5 \times 1.75 \mathrm{arcmin}^{2}$. The detector arrays are made of 8 and 2 filled bolometer matrices with $16 \times 16$ pixels each, respectively. The arrays operate at $\approx 285 \mathrm{mK}$ provided by a ${ }^{3} \mathrm{He}$ sorption cooler with a hold time of $57.8 \mathrm{~h}$, if the bolometers are biased all the time after the cooler recycling.

The principal science observation mode with the PACS photometer is the scan map with the telescope scanning along parallel legs covering the map are 11 The nominal scan map speed is $201 / \mathrm{s}$. In most cases both an in-scan and a cross-scan map were taken in order to reduce the striping in the combined maps and for getting a better handle on extended structures. Two flavors of scan maps were used:

1) Large scan maps with scan angle orientations of usually 45 and $135^{\circ}$. The scan leg separation is freely selectable ranging from relatively wide separations ensuring homogeneous coverage of large fields to narrow separations for high redundancy ${ }^{2}$

2) Mini scan maps for dedicated point/compact source photometry have only extensions in the order of about $3 \mathrm{arcmin}$, scan angle orientations of 70 and $110^{\circ}$ and narrow scan leg separation of a few arcsec. This mode is also used for the flux calibration ${ }^{3}$

An alternative observation mode, the chop/nod point source photometry, has been maintained over the full mission for flux calibration consistency checking and in support of observatory pointing calibration. Its flux calibration is described in this issue [14].

\section{Basic Photometric Calibration Strategy}

The physical quantity which determines the bolometer's absolute photometric calibration is its responsivity R. R, in units [V/W], is the ratio of the measured output signal $\mathrm{U}_{\text {sig }}$ produced by the infalling far-infrared radiation power $\mathrm{F}_{\text {photband }}$, hence

$$
U_{\text {sig }}=R \times F_{\text {photband }}
$$

1 (for further reference see http://herschel.esac.esa.int/Docs/PACS/html/pacs_om.html)

2 http://herschel.esac.esa.int/twiki/pub/Public/PacsAotReleaseNotes//PACS_ScanMap_ReleaseNote_23Feb2010.pdf

3 http://herschel.esac.esa.int/twiki/pub/Public/PacsCalibrationWeb/PhotMiniScan_ReleaseNote_20101112.pdf 
With a source SED, conventionally expressed as $\mathrm{f}_{v, s}(\lambda)[\mathrm{Jy}]$ in the FIR, and the relation $f_{\lambda}=\frac{c}{\lambda^{2}} f_{v}$, the flux in a PACS photometer band can be expressed as

$$
F_{\text {photband }}[W]=T A \int_{\lambda_{1}}^{\lambda_{2}} \frac{c}{\lambda^{2}} f_{v, s}(\lambda) S(\lambda) d \lambda
$$

with $\mathrm{T}$ being the product of reflection losses by the optical mirrors, A being the effective telescope area and $S(\lambda)$ being the relative photometer system response of the PACS photometer band.

For the PACS photometer, the convention is, that the flux density at the reference wavelength $\mathrm{f}_{v, s}\left(\lambda_{0}\right)$ is determined for the reference SED $\mathrm{f}_{v, 1}=v^{-1}$, i.e. $f_{v, 1}(\lambda)=\frac{v_{0}}{v} f_{v, 1}\left(\lambda_{0}\right)=$ $\frac{\lambda}{\lambda_{0}} f_{v, 1}\left(\lambda_{0}\right)$, hence

$$
\begin{gathered}
F_{\text {photband }}[W]=T A f_{v, 1}\left(\lambda_{0}\right) \frac{c}{\lambda_{0}} \int_{\lambda_{1}}^{\lambda_{2}} \frac{1}{\lambda} S(\lambda) d \lambda \\
f_{v, 1}\left(\lambda_{0}\right)=\frac{F_{\text {photband }}}{T A \frac{c}{\lambda_{0}} \int_{\lambda_{1}}^{\lambda_{2}} \frac{1}{\lambda} S(\lambda) d \lambda}=F_{\text {photband }} C_{\text {conv }}
\end{gathered}
$$

with

$$
C_{\mathrm{conv}}=\frac{1}{T A \frac{c}{\lambda_{0}} \int_{\lambda_{1}}^{\lambda_{2}} \frac{1}{\lambda} S(\lambda) d \lambda}=\frac{1}{T A \Delta v_{0}}
$$

and effective bandwidth

$$
\Delta v_{0}=\frac{c}{\lambda_{0}} \int_{\lambda_{1}}^{\lambda_{2}} \frac{1}{\lambda} S(\lambda) d \lambda
$$

If the source $\operatorname{SED~} \mathrm{f}_{v, s}(\lambda)$ is known, the true photometer source flux is determined as

$$
f_{v, s}\left(\lambda_{0}\right)=\frac{f_{v, 1}\left(\lambda_{0}\right)}{K_{c c}}
$$

with $\mathrm{K}_{c c}$ being the appropriate color correction factor as described in [17] and in the document "PACS Photometer Passbands and Colour Correction Factors for various source SEDs", PICC-ME-TN-038

This means that

$$
R \propto \frac{1}{F_{\text {photband }}} \propto \frac{C_{\text {conv }}}{f_{v, 1}\left(\lambda_{0}\right)} \propto \frac{1}{T \times A \times \Delta v_{0} \times f_{v, s}\left(\lambda_{0}\right) \times K_{c c}}
$$

hence the responsivity $\mathrm{R}$ can be calibrated, too, by flux density measurements of celestial standards.

The responsivity of the bolometer arrays depends on their operating temperature, voltage bias, and optical loading (see Sec. 6). R was thoroughly characterized in the lab (cf. [2]), and the ground-based calibration was used to estimate the initial in-flight responsivity at the beginning of the mission when the telecope foreground emission had stabilized. For in-flight

\footnotetext{
4 http://herschel.esac.esa.int/twiki/pub/Public/PacsCalibrationWeb/cc_report_v1.pdf
} 
calibration only a relative update in the form of

$$
\frac{R_{\text {new }}}{R_{\text {old }}}=\frac{f_{v, s}\left(\lambda_{0}\right)}{f_{v, \text { standardmodel }}\left(\lambda_{0}\right)}
$$

was necessary, by using the ratio of the measured flux to the celestial standard star model flux, when calibrating $f_{v, s}\left(\lambda_{0}\right)$ with $\mathrm{R}_{\text {old }}$.

In practice, the update of $\mathrm{R}$ did not rely on one single flux measurement alone, but on a set of measurements on several standards to minimize systematic effects

$$
\frac{R_{\text {new }}}{R_{\text {old }}}=\frac{1}{n} \sum_{i=1}^{n} \frac{f_{V, s, i}\left(\lambda_{0}\right)}{f_{V, \text { standardmodel }, i}\left(\lambda_{0}\right)}
$$

so that $\frac{1}{n} \sum_{i=1}^{n} \frac{f_{V, s, i}\left(\lambda_{0}\right)}{f_{V, \text { standardmodel }, i}\left(\lambda_{0}\right)}=1$ by applying $\mathrm{R}_{\text {new }}$.

This scheme applies, when more standard star measurements become available, when additional systematic instrument effects altering the signal level are corrected for, when changes in the encircled energy fraction alter the resulting flux (see below) or when new standard star models become available.

Note, that $\mathrm{R}$ is a global value for the whole detector array. Individual pixel-to-pixels variations are accounted for by the flat-field $\mathrm{ff}_{p i x}$.

$\mathrm{R}$ is not a constant. It depends on temperature and the total flux load, hence $\mathrm{R}=\mathrm{R}(\mathrm{T}$, $\mathrm{B}_{\text {totalflux }}$ ), as will be shown later.

The standard evaluation procedure for flux calibration measurements is aperture photometery. Hence, not the the total source flux is measured, but only the fraction inside the measurement aperture. For the derivation of the total measured source flux a correction for the encircled energy fraction (EEF 5 , see Table 2 inside the aperture must be performed:

$$
f_{v, 1}\left(\lambda_{0}\right)=\frac{f_{v, 1}^{\text {aperture }}\left(\lambda_{0}\right)}{f_{E E F}}
$$

\section{Photometric Calibration Standards}

As prime flux reference, models of the photospheric emission of late type giants are used [6]. This type of stars was already used as absolute calibrators for earlier IR space missions (e.g. IRAS: [20], ISO: [3], Spitzer: [8]). The flux regime they cover is inside the linear flux behaviour of the PACS bolometers. Ideally, stars of different stellar types would be used to prevent systematic uncertainties from the modeling. Unfortunately only the late type giants are bright enough at far infrared wavelengths to be observed at high signal to noise. One star, Sirius ( $\alpha \mathrm{CMa}$ ), was the only A type exception, but unfortunately had to be dropped from our set of primary flux calibrators as will be discussed in 3.2 Models of the atmospheres of the giant planets Uranus and Neptune [12] are equally accurate, however these sources are already in the non-linear flux regime of PACS. Asteroids are about to be established as independent prime FIR flux calibrators ( [13], this volume). The five fiducial stellar standards $\alpha$ Boo, $\alpha$ Cet, $\alpha$ Tau, $\beta$ And and $\gamma$ Dra were observed repeatedly during the

\footnotetext{
5 http://herschel.esac.esa.int/twiki/pub/Public/PacsCalibrationWeb/bolopsf_20.pdf
} 
mission for absolute calibration and to monitor the system stability (for this $\gamma \mathrm{Dra}$, which was visible during the whole mission, was observed on a monthly basis). We also monitored the photometric system using the Calibration Blocks (CalBlocks). They track the response several times a day throughout the mission (at least one Calblock per OBSID) and show the high stability of the photometric system with high accuracy (see [11]).

\subsection{Summary of mini scan-map observations}

All measurements were taken as part of one of the following calibration programmes: "RPPhotFlux_321B”, "RPPhotFlux_324B”, "RPPhotFlux_321D”, "PVPhotAOTVal_514P”. All observations were taken in medium scan speed with 20 " $/ \mathrm{s}$, in "high gain" and only one single repetition of each scan-map. All measurements were preceded by a 30-s calibration measurement on the PACS internal calibration sources (see [11])The detailed information on the observation of the individual fiducial stars can be found in Tables 5, 6, 7, 8, and 9 . All observations taken with the PACS Photometer from Operational Day (OD)\#1375 (16 February 2013) onwards are affected by a serious anomaly detected in one of the two red channel subarrays (matrix 9). Data taken in ODs\#1375 and \#1376 from this subarray were found to be uniformly saturated. Recovery activities taking place in subsequent days were unsuccessful. Further observations taken in OD\#1380 confirmed the problem. It was also confirmed that the other red channel subarray (matrix 10), was working correctly. This results essentially in a degradation of a factor 1.4 in sensitivity and reduced spatial coverage of all the images taken in the 160 micron band, ranging from some reduction of the map area, to a loss of up to one third of the map area in the most extreme cases. Altogether six calibration observations (taken after OD\#1376) were affected by this anomaly. Fortunately the layout of our observations and the flux level of the sources ensured that the loss in sensitivity did not influence the photometry.

\subsection{Model fluxes}

The theoretical spectra of candidate stellar calibrators in the far infrared were generated using the MARCS stellar atmosphere code ([9], [10]) and the TURBOSPECTRUM synthetic spectrum code $([16])$ and are presented in [6]. The stellar parameters and their uncertainties were derived by [4]. The line lists used in the spectrum calculations and the model uncertainties are discussed in [5], where it is estimated that the uncertainty of the models in the PACS wavelength range is $\approx 5 \%$. The absolute flux calibration is based on Selby's ([21]) K-band photometry, the zero-point is determined on the basis of an ideal 'Vega', i.e. the K-band photometry of Vega is corrected for a flux excess of $1.29 \%$ (cf. [1]). The determined Selby K-band zeropoint is $4.0517 \times 10^{-10} \mathrm{~W} / \mathrm{m}^{2} / \mu \mathrm{m}$. This and more detailed information on the individual stars can be found in the headers of the fits files containing the theoretical spectra used for the PACS flux calibration. The files can be found at ftp.ster.kuleuven.be/dist/pacs/calsources/ or ftp.sciops.esa.int and will be provided in the Herschel archive.

As the stars can show excess flux in the far infrared wavelengths, due to either debris disks for early type dwarfs or in the case of late type giants, by a chromosphere or ionised wind, it is important to investigate whether we can rule out the existence of such excesses for our candidate calibrators. [6] present observations in the sub-mm up to $\mathrm{cm}$ wavelength range for nine late type giant stars, of which seven were in our original list of candidate calibrators. 
Table 1 Information on the selected fiducial stars. Monochromatic flux densities at 70.0, 100.0 and $160.0 \mu \mathrm{m}$ are given. The stellar temperatures are taken from [4] and the fluxes are based on models published in [6].

\begin{tabular}{|c|c|c|c|c|c|c|c|c|c|c|}
\hline \multirow[b]{2}{*}{ HR } & \multirow[b]{2}{*}{ HD } & \multirow[b]{2}{*}{ HIP } & \multirow[b]{2}{*}{ ID } & \multirow[b]{2}{*}{ RA (J2000) } & \multirow[b]{2}{*}{$\operatorname{Dec}(\mathrm{J} 2000)$} & \multirow[b]{2}{*}{ SpType } & \multirow{2}{*}{$\begin{array}{l}\text { Temp } \\
{[\mathrm{K}]}\end{array}$} & \multicolumn{3}{|c|}{ Model flux [mJy] } \\
\hline & & & & & & & & $70 \mu \mathrm{m}$ & $100 \mu \mathrm{m}$ & $160 \mu \mathrm{m}$ \\
\hline 337 & 6860 & 5447 & $\beta$ And & 01:09:43.9236 & $+35: 37: 14.008$ & MOIII & 3880 & 5594 & 2737 & 1062 \\
\hline 911 & 18884 & 14135 & $\alpha \mathrm{Cet}$ & 03:02:16.8 & $+04: 05: 24.0$ & M1.5IIIa & 3740 & 4889 & 2393 & 928 \\
\hline 1457 & 29139 & 21421 & $\alpha$ Tau & $04: 35: 55.2387$ & $+16: 30: 33.485$ & K5III & 3850 & 14131 & 6909 & 2677 \\
\hline 5340 & 124897 & 69673 & $\alpha$ Boo & $14: 15: 39.6720$ & $+19: 10: 56.677$ & K1.5III & 4320 & 15434 & 7509 & 2891 \\
\hline 6705 & 164058 & 87833 & $\gamma$ Dra & 17:56:36.3699 & $+51: 29: 20.022$ & K5III & 3960 & 3283 & 1604 & 621 \\
\hline
\end{tabular}

These observations were made in preparation of the Herschel mission and performed on several ground based telescopes. Also Sirius, a A1V star, was studied, but not included in the [6] paper as this only considered $\mathrm{K}$ and $\mathrm{M}$ giants. Although in seven out of nine studied giants an excess was detected, it was found that for the eight stars, selected as prime flux calibrators for the PACS photometer, the excess only started beyond the PACS wavelength range.

Based on early analysis of Herschel observations of potential calibration stars we decided to use only the following 5 fiducial stars for the final analysis: $\beta$ And, $\alpha$ Cet, $\alpha$ Tau, $\alpha$ Boo, $\gamma$ Dra. The three other potential calibrators were discarded ( $\alpha$ CMa, $\beta$ Peg, $\beta$ UMi). In the case of these three stars the fluxes deviated from the models by more than $10 \%$ in at least one PACS band. For $\beta$ UMi no Selby K-band photometry was available for the absolute calibration and only a less accurate $(\sim 10 \%)$ Johnson $\mathrm{K}$ band from [7] could be used. [19] showed that $\beta$ Peg is variable in the mid-IR bands by about $10 \% . \alpha$ CMa shows about $20 \%$ excess at $160 \mu \mathrm{m}$. The underlying cause of the discrepancy is still unclear and is under investigation.

The monochromatic flux densities based on at 70.0, 100.0 and $160.0 \mu \mathrm{m}$ for the five finally selected stars are given in Table 1

\section{Determination of flux densities}

4.1 Data reduction and calibration

\subsubsection{Pre-processing}

The data reduction was done with software version "hcss.dp.pacs-11.0.2600", calibration tree No. 58 and the following steps, parameters and calibration file versions:

- Flagging of bad pixels (badPixelMask: FM, 6)

- Flagging of saturated pixels (clSaturationLimits: FM, 1; satLimits: FM, 2)

- Conversion digital units to Volts

- Adding of pointing and time information (we used the updated pointing product for observations taken between OD 320 and OD 761)

- Response calibration (responsivity: FM, 7)

- Flat fielding (flatField: FM, 4) 


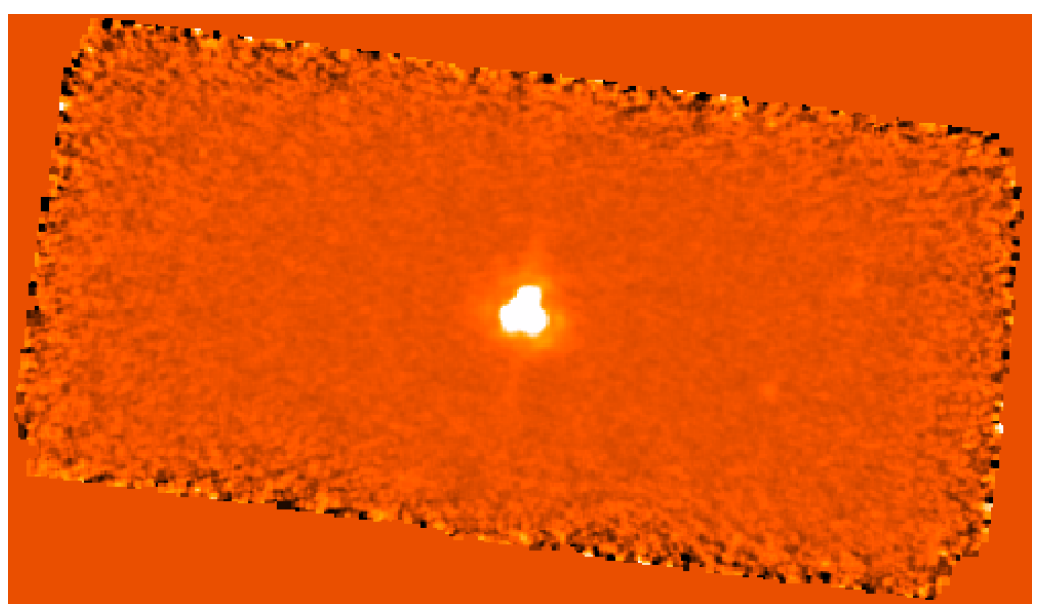

Fig. 1 Mini scanmap of $\alpha$ Tau obtained at $70 \mu \mathrm{m}$ as a typical example for a flux calibration measurement.

- Frame selection based on scan speed $( \pm 10 \%$ of the nominal speed)

$($ frames $=$ filterOnScanSpeed (frames, limit=10.0, copy=None)

- Second level deglitching

- Save the observation context into a fits-file (including still all frames)

\subsubsection{Post-processing}

The purpose of post processing is to eliminate the $1 / \mathrm{f}$ noise of the detector using high-pass filtering of the data. The process includes two steps. The purpose of the first step is to locate and mask the source using the level 2 product and a circular mask with a size of $25^{\prime \prime}$, while the second steps includes the high-pass filtering of the data.

- Set on-target flag for all frames to 'true'

- Set a circular mask around the position of the source $\left(\mathrm{r}=25^{\prime \prime}\right)$

- High-pass filtering with a hp-filter width of 15, 20, 35 in blue, green and red band respectively (adjusted to the FWHM of the corresponding PSFs, see Section 4.1.3 for details)

- Merging frames (join) of both scan directions (usually $70^{\circ}$ and $110^{\circ}$ scan angles in instrument frame: approx. along the diagonals of the bolometer array)

- Frame selection based on scan speed $( \pm 10 \%$ of the nominal speed $)$

- Final projection of all data with photProject(), using the default pixel fraction $($ pixfrac $=1.0)$ and reduced pixel sizes of $1.1^{\prime \prime}, 1.4^{\prime \prime}, 2.1^{\prime \prime}$

- Save final map as fits-file (see Fig. 1) 


\subsubsection{Determination of the high-pass filter width}

We determined the high-pass filter width in each band to account for the difference in their FWHM of the PSF and to make sure that the mask is large enough so that the high-pass filtering does not remove flux from the source. All the data used in the calibration was taken at $20^{\prime \prime} / \mathrm{s}$. The bolometer data are taken with $40 \mathrm{~Hz}$ with an onboard averaging of 4 frames in both channels in the PACS prime mode leading to a data rate of $10 \mathrm{~Hz}$ in the downlink. The FWHM of a point-source is about 5.6" in the blue band, $6.8^{\prime \prime}$ in the green band and $11.3^{\prime \prime}$ (average values for $20^{\prime \prime} / \mathrm{s}$ scan speed), so in a signal time-line for a given pixel a central hit of the source has therefore the following width: $5.6^{\prime \prime} / 20^{\prime \prime} / \mathrm{s}=0.28 \mathrm{~s}$ or 2.8 frames in blue, $6.8^{\prime \prime} / 20^{\prime \prime} / \mathrm{s}=0.34 \mathrm{~s}$ or 3.4 frames in green, $11.3^{\prime \prime} / 20^{\prime \prime} / \mathrm{s}=0.565 \mathrm{~s}$ or 5.65 frames in red. The values 15, 20, and 35 for the high-pass-filter parameter correspond to 31, 41, and 71 frames per filter width $(2 \times$ hpf-parameter +1$)$. Considering the scanning speed of $20^{\prime \prime} / \mathrm{s}$ and a $10-\mathrm{Hz}$ sampling, these frame numbers translate to $62^{\prime \prime}, 82^{\prime \prime}$, and $142^{\prime \prime}$ filter width at 70,100 , and $160 \mu \mathrm{m}$, respectively. The ratios between FWHM and high-pass filter width are 2.8/31 $=0.09$ in blue, $3.4 / 41=0.08$ in green and 5.65/71 $=0.08$ in red. These ratios are very similar in the three bands and at a very conservative level so that the high-pass filtering is not affecting the source flux with the appropriate masking. 


\subsection{Photometry}

\subsubsection{Source flux determination}

We determined the flux using aperture photometry with aperture sizes of $12^{\prime \prime}, 12^{\prime \prime}, 22^{\prime \prime}$ in blue, green, and red, respectively (centering the aperture on the flux peak). These values are based on an analysis of the influence of the high-pass filter width on aperture photometry [18]. It shows that a small aperture is better, as long as the aperture correction is applied. The larger the aperture, the more the photometry will depend on the filter width. These apertures are chosen such that the uncertainty because of the high-pass filter width is less than $1 \%$. This approach has the advantage that the results can be relatively independent of high-pass filter width. Note, that at lower flux levels one should select significantly smaller aperture sizes for better $\mathrm{S} / \mathrm{N}$.

For background subtraction, we selected an annulus between $35^{\prime \prime}$ and $45^{\prime \prime}$. We applied aperture corrections of $0.802,0.776$, and 0.817 for blue, green and red filters respectively (see Table 2 ), to account for the flux outside the aperture. These values are based on very large maps (extending to $15^{\prime}$ from a bright source) indicating that about $10 \%$ more flux is in the outermost wings of the PSFs beyond $60^{\prime \prime}$. Although the wings of the PSF extending far outside the outer limit of our background annulus there is no leftover flux from the PSF affecting our photometry because the high-pass filtering effectively removes the source flux from the image outside the boundary of our mask $\left(r=25^{\prime \prime}\right)$ while it leaves the flux unaffected inside. Detailed investigation of the effect of the background annulus on the final photometry of the standard stars show that our measurements are affected by less than $0.1 \%$ by the leftover flux of the PSF wings within the background annulus. As an illustration, Fig. 2 shows the photometry of $\alpha$ Boo (the brightest of our five fiducial stars; see Table 1 at $70 \mu \mathrm{m}$ using different background annuli. The horizontal axis corresponds to the inner edge while the different points at the same inner radius value refer to different outer radii. To guide the reader we show the inner radius of the annulus used for the photometry of the standards (vertical line) and the $\pm 0.1 \%$ limits (horizontal lines) with respect to the photometry using the outermost annulus (between $60^{\prime \prime}$ and $80^{\prime \prime}$ ). It is clear from the figure that the placement and the size of the background annulus does not affect the photometry by more than $0.1 \%$ outside of the $30^{\prime \prime}$ inner radius. At smaller inner radii however the effect is noticeable and needs to be corrected. The PACS ICC is currently working on the new calibration file that corrects this effect in HIPE.

Note, that the previous values, connected to earlier versions of PSFs (and assuming that there is no flux beyond $60^{\prime \prime}$ ) and to the same aperture sizes, were: 0.886 (blue band, $12^{\prime \prime}$ aperture radius), 0.866 (green band, $12^{\prime \prime}$ aperture radius), and 0.916 (red band, $22^{\prime \prime}$ aperture radius). These values were correct for responsivity calibration file (FM,5), but are not correct for later version: 7

\subsubsection{Source flux uncertainty determination}

We determined the flux uncertainty of the calibration source measurements in two different ways.

\footnotetext{
6 seehttp://herschel.esac.esa.int/twiki/pub/Public/PacsCalibrationWeb/bolopsf_20.pdf for an in depth analysis of the PACS PSF and the calculation of the encircled energy fractions

7 see http://herschel.esac.esa.int/twiki/pub/Public/PacsCalibrationWeb/PhotMiniScan_ReleaseNote_20101112.pdf
} 


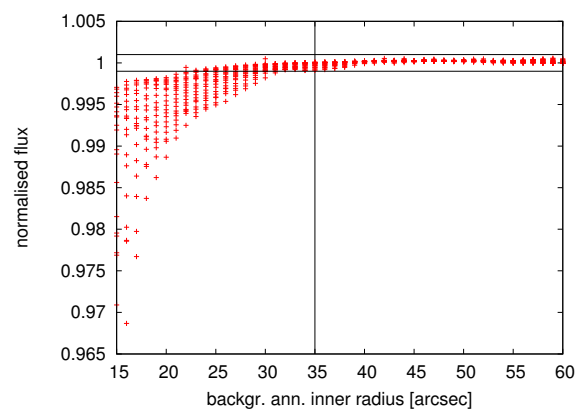

Fig. 2 Photometry of $\alpha$ Boo using different background annuli. The fluxes are normalized to the value measured using the annulus between $60^{\prime \prime}$ and $80^{\prime \prime}$. The vertical line shows the inner radius of the background annulus used for our photometry, while the horizontal line designate the $\pm 0.1 \%$ limit.

1 By performing aperture photometry on the background annulus. We used the same aperture sizes as for the source photometry. We distributed six apertures evenly along the background annulus, measured the flux without background subtraction and determined the standard deviation of the measured values after aperture correction.

2 By estimating the noise inside the annulus used for background subtraction in all three bands (signal r.m.s. inside the selected sky annulus). Note that the noise per pixel in the final maps depends on the selected pixel size, the high-pass filter width and also the "drop size". We only used one set of fixed values for the high-pass filtering ( 15 in blue, 20 in green and 35 in red band) and the default drop size (pixfrac $=1.0$ ), the so called correlated noise in the final maps is therefore a consequence of the regridding the data from the pixel values of the detector array into maps projected on the sky. This modifies the measured SNR (signal-to-noise ratio). In particular, if small map pixels are selected, the measured SNR of the integrated flux can appear to be much higher than the true value. The correction for the correlated noise (rms-noise values have to be divided by these factors) amounts to:

- blue band: $1.00 \times(\text { pixsize } / 3.2)^{1.78}$; here: $1.00 \times(1.1 / 3.2)^{1.78}=0.1495$

$(\rightarrow$ noise increases by a factor of 6.7 )

- green band: $1.01 \times(\text { pixsize } / 3.2)^{1.70}$; here: $1.01 \times(1.4 / 3.2)^{1.70}=0.2477$

$(\rightarrow$ noise increases by a factor of 4.0$)$

- red band: $1.02 \times(\text { pixsize } / 6.4)^{1.51}$; here: $1.02 \times(2.1 / 6.4)^{1.51}=0.1896$

$(\rightarrow$ noise increases by a factor of 5.3)

The correction applied to the photometric uncertainty was established in the following way. Using a template observation, the rms of the pixel-to-pixel flux variations was calculated for each fully calibrated Level 1 detector frame. The ratio between the theoretical final photometric uncertainty by assuming a Gaussian error propagation of the Level 1 frames and the value measured in the final Level 2 was obtained for varying map pixel sizes and default drizzling drop sizes. This set of ratios is used for correcting the photometric uncertainties for the unaccounted correlated noise. Finally we determined the error of the source flux using the following formula:

corrected, r.m.s. values $\times \frac{\sqrt{(\text { number of map pixel inside the specified aperture })}}{\text { aperture correction }}$ 
Table 2 Encircled Energy Fraction (EEF) as a function of circular aperture radius for the three PACS filter bands. Valid for responsivity calibration file version FM, 7 . See http://herschel.esac.esa.int/twiki/pub/Public/PacsCalibrationWeb/bolopsf_20.pdf for an in depth analysis of the PACS PSF and the calculation of the encircled energy fractions

\begin{tabular}{|cccc|cccc|}
\hline radius & \multicolumn{2}{c|}{ Encircled Energy } & Fraction & \multicolumn{3}{c|}{ radius } & \multicolumn{3}{c|}{ Encircled Energy } & Fraction \\
{$[\prime \prime]$} & blue & green & red & {$[\prime \prime]$} & blue & green & red \\
\hline 2 & 0.192 & 0.141 & 0.060 & 32 & 0.896 & 0.891 & 0.861 \\
3 & 0.353 & 0.278 & 0.127 & 33 & 0.898 & 0.893 & 0.864 \\
4 & 0.487 & 0.413 & 0.209 & 34 & 0.900 & 0.895 & 0.867 \\
5 & 0.577 & 0.521 & 0.298 & 35 & 0.902 & 0.896 & 0.870 \\
6 & 0.637 & 0.595 & 0.384 & 36 & 0.904 & 0.898 & 0.873 \\
7 & 0.681 & 0.641 & 0.461 & 37 & 0.905 & 0.900 & 0.876 \\
8 & 0.719 & 0.673 & 0.527 & 38 & 0.907 & 0.902 & 0.879 \\
9 & 0.751 & 0.700 & 0.579 & 39 & 0.908 & 0.903 & 0.882 \\
10 & 0.774 & 0.727 & 0.619 & 40 & 0.910 & 0.905 & 0.884 \\
11 & 0.791 & 0.753 & 0.649 & 41 & 0.911 & 0.906 & 0.886 \\
12 & 0.802 & 0.776 & 0.673 & 42 & 0.913 & 0.908 & 0.888 \\
13 & 0.812 & 0.795 & 0.694 & 43 & 0.914 & 0.909 & 0.890 \\
14 & 0.820 & 0.808 & 0.712 & 44 & 0.916 & 0.910 & 0.892 \\
15 & 0.829 & 0.818 & 0.729 & 45 & 0.917 & 0.912 & 0.894 \\
16 & 0.837 & 0.826 & 0.746 & 46 & 0.919 & 0.913 & 0.896 \\
17 & 0.845 & 0.832 & 0.761 & 47 & 0.920 & 0.914 & 0.897 \\
18 & 0.852 & 0.837 & 0.776 & 48 & 0.921 & 0.915 & 0.899 \\
19 & 0.858 & 0.842 & 0.789 & 49 & 0.922 & 0.917 & 0.901 \\
20 & 0.863 & 0.847 & 0.800 & 50 & 0.924 & 0.918 & 0.902 \\
21 & 0.867 & 0.852 & 0.809 & 51 & 0.925 & 0.919 & 0.904 \\
22 & 0.870 & 0.857 & 0.817 & 52 & 0.926 & 0.920 & 0.905 \\
23 & 0.874 & 0.863 & 0.824 & 53 & 0.927 & 0.921 & 0.906 \\
24 & 0.877 & 0.867 & 0.830 & 54 & 0.929 & 0.922 & 0.908 \\
25 & 0.880 & 0.872 & 0.835 & 55 & 0.930 & 0.923 & 0.909 \\
26 & 0.883 & 0.876 & 0.839 & 56 & 0.931 & 0.924 & 0.910 \\
27 & 0.885 & 0.879 & 0.843 & 57 & 0.932 & 0.925 & 0.911 \\
28 & 0.888 & 0.882 & 0.847 & 58 & 0.933 & 0.926 & 0.912 \\
29 & 0.890 & 0.885 & 0.850 & 59 & 0.935 & 0.928 & 0.913 \\
30 & 0.892 & 0.887 & 0.854 & 60 & 0.936 & 0.929 & 0.914 \\
31 & 0.894 & 0.889 & 0.857 & 61 & 0.937 & 0.929 & 0.915 \\
\hline
\end{tabular}

Almost always the first method resulted in larger values so we accepted the values from this method as the final error estimate. Note, that the source flux uncertainties in case of the fiducial stars are very small. SNR values are well above 100 . The error bars throughout this report are therefore in most cases smaller than the symbol sizes.

\subsubsection{Colour correction}

The colour correction values for the fiducial stars $(1.016,1.033,1.074)$ were derived from a $4000 \mathrm{~K}$ black body, very close to the effective temperature of the stars (see Table. 1), ranging between $3740 \mathrm{~K}$ and $4320 \mathrm{~K}$. We repeated now the calculation using the official model template files (see section 3.2 and could confirm the tabulated values in the blue and red band. In the green band we found a difference of $0.1 \%$ (1.034) when using the full stellar templates instead of a $4000 \mathrm{~K}$ black body. No difference between the K- and M-giants are seen on the per mille level. In the final error budget this small deviation can be neglected and the colour correction is not contributing to the systematic differences between $\mathrm{K}$ - and M-giants seen in the calibrated flux densities of the 5 fiducial stars. 
The following colour correction factors have been used to obtain monochromatic flux densities and uncertainties at 70.0, 100.0, $160.0 \mu \mathrm{m}$ :

\begin{tabular}{lll}
\hline $70.0 \mu \mathrm{m}$ & $100.0 \mu \mathrm{m}$ & $160.0 \mu \mathrm{m}$ \\
\hline 1.016 & 1.033 & 1.074 \\
\hline
\end{tabular}

\section{End of mission calibration status}

The results for the fiducial stars we obtained based on the responsivity calibration file "FM, 7 " are listed in Table 3 The derived mean values show that the currently available calibra-

Table 3 Observed and calibrated ("FM, 7") monochromatic flux densities at 70, 100, $160 \mu$ m divided by the corresponding current model predictions for all 5 fiducial stars.

\begin{tabular}{lrrrrrrrrr}
\hline \multirow{2}{*}{$\begin{array}{l}\text { Target } \\
\text { name }\end{array}$} & \multicolumn{3}{c}{ blue obs/model } & \multicolumn{3}{c}{ green obs/model } & \multicolumn{2}{c}{ red obs/model } \\
& no. & mean & stdev & no. & mean & stdev & no. & mean & stdev \\
\hline$\beta$ And & 6 & $\mathbf{1 . 0 1 7}$ & 0.007 & 6 & $\mathbf{1 . 0 1 7}$ & 0.007 & 6 & $\mathbf{0 . 9 9 1}$ & 0.010 \\
$\alpha$ Cet & 7 & $\mathbf{1 . 0 1 3}$ & 0.006 & 7 & $\mathbf{1 . 0 1 3}$ & 0.005 & 7 & $\mathbf{1 . 0 2 0}$ & 0.015 \\
$\alpha$ Tau & 7 & $\mathbf{0 . 9 6 8}$ & 0.013 & 7 & $\mathbf{0 . 9 6 9}$ & 0.015 & 8 & $\mathbf{0 . 9 6 7}$ & 0.015 \\
$\alpha$ Boo & 7 & $\mathbf{0 . 9 8 6}$ & 0.015 & 7 & $\mathbf{0 . 9 9 3}$ & 0.014 & 7 & $\mathbf{1 . 0 0 1}$ & 0.021 \\
$\gamma$ Dra & 54 & $\mathbf{0 . 9 8 4}$ & 0.009 & 11 & $\mathbf{0 . 9 9 0}$ & 0.010 & 60 & $\mathbf{1 . 0 1 0}$ & 0.029 \\
\hline \multicolumn{2}{l}{ mean/stdev } & $\mathbf{0 . 9 9 4} \pm \mathbf{0 . 0 2 1}$ & & $\mathbf{0 . 9 9 7} \pm \mathbf{0 . 0 1 9}$ & & $\mathbf{0 . 9 9 8} \pm \mathbf{0 . 0 2 0}$ \\
\hline
\end{tabular}

tion files with the aperture correction provide a very accurate point source flux calibration. The intrinsic accuracy of the photometry is around $2 \%$ in all three filter bands. Considering the $5 \%$ uncertainty of the models of the fiducial stars, the final photometric calibration uncertainty is less than $7 \%$.

Figure 3 shows the measured over model flux ratios for all individual measurements at $70 \mu \mathrm{m}$ (upper left), $100 \mu \mathrm{m}$ (upper right) and $160 \mu \mathrm{m}$ (lower left). There is one measurement of $\gamma$ Dra at $70 \mu \mathrm{m}$ that deviates from the locus of the other measurements on the same object. The measurement occurred on OD 1308 when a large pointing offset was introduced by not updating the $\mathrm{S} / \mathrm{C}$ velocity vector by MOC during the DTCP, so as a precaution we leave this point out of the analysis.

It is interesting to note that while the individual scatter per source is clearly the largest in the red band the standard deviation of the averages are rather similar. Also in the blue and green bands the fiducial star measurement are systematically different (e.g. the flux ratio of the 2 M-type giants ( $\beta$ And and $\alpha$ Cet) are larger by about 3-4\% than the flux ratio of the three K-type giants). This shows that uncertainty of the calibration is dominated by the discrepancy of the model fluxes.

The numbers in Table 3 show that the calibrations are equally good at all wavelengths. So the relative photometric accuracy between the different PACS bands is better than $1 \%$.

Figure 4 shows the results summarised in Table 3 

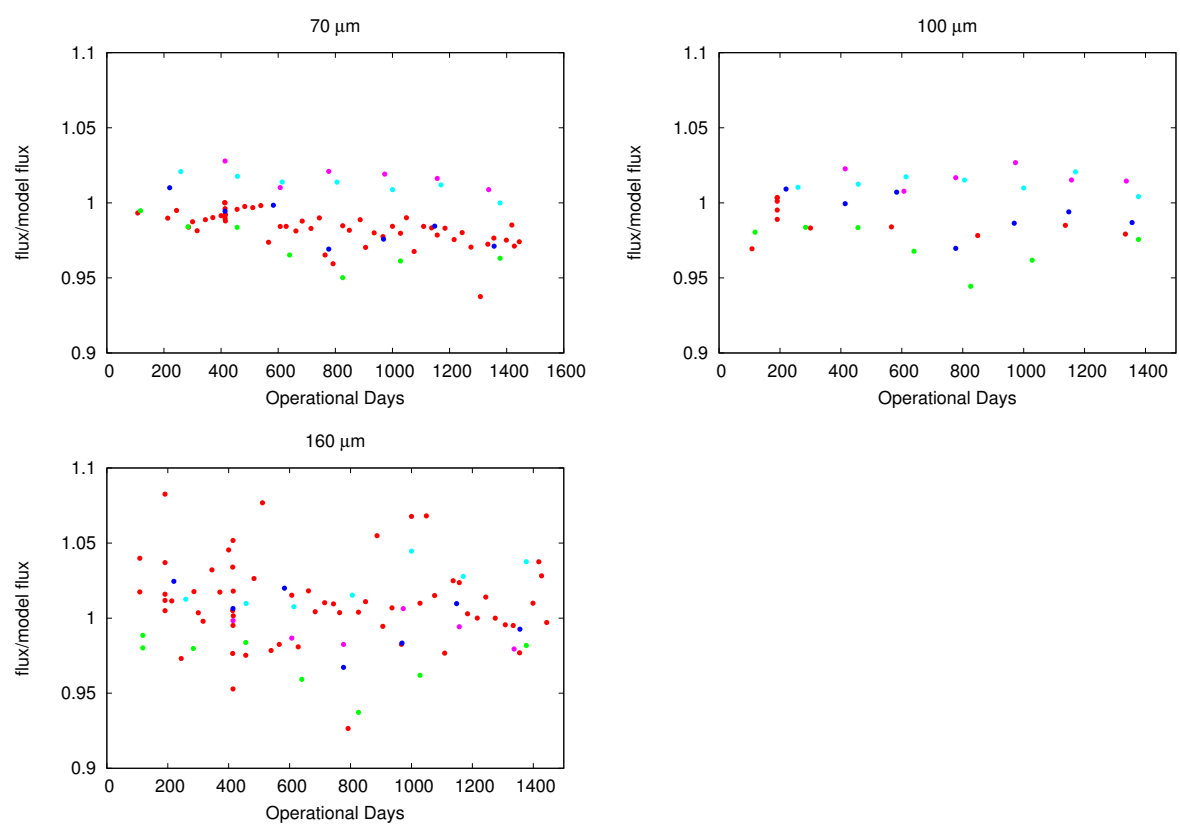

Fig. 3 Measured over model flux ratios of the five fiducial standards at $70 \mu \mathrm{m}$ (upper left), $100 \mu \mathrm{m}$ (upper right) and $160 \mu \mathrm{m}$ (lower left). The different colours designate different objects. Red: $\gamma$ Dra; green: $\alpha$ Tau; blue: $\alpha$ Boo; magenta: $\beta$ And; cyan: $\alpha$ Cet. We follow this convention throughout the paper.

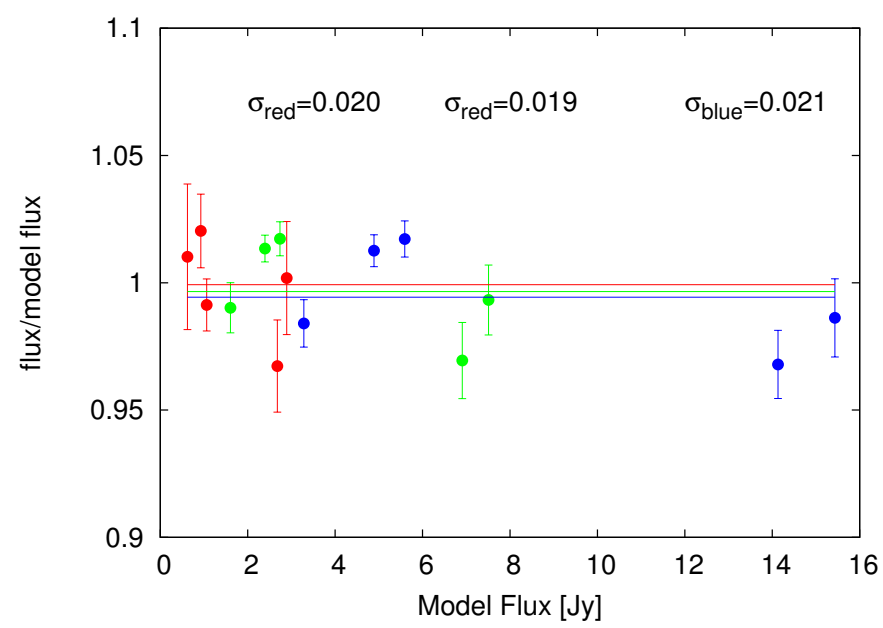

Fig. 4 Final result of the calibration. Red dots: $160 \mu \mathrm{m}$; green dots: $100 \mu \mathrm{m}$; blue dots: $70 \mu \mathrm{m}$. The red, green and blue lines represent the average values at $160 \mu \mathrm{m}, 100 \mu \mathrm{m}$, and $70 \mu \mathrm{m}$ respectively. The error bars represent the standard deviation of the individual set of measurements (each filter/object combination) 

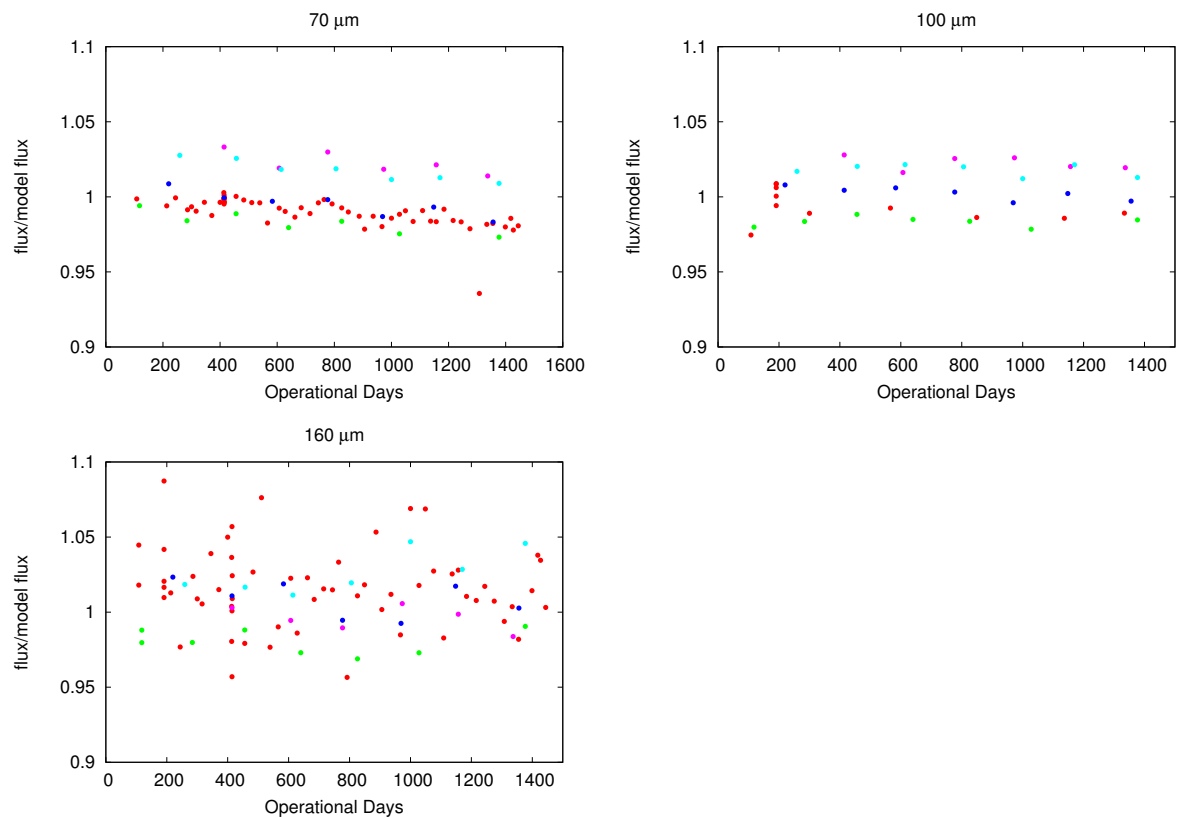

Fig. 5 Measured over model flux ratios of the five fiducial standards at $70 \mu \mathrm{m}$ (upper left), $100 \mu \mathrm{m}$ (upper right) and $160 \mu \mathrm{m}$ (lower left) after applying the correction for the evaporator temperature.

\section{Characterization of instrumental effects affecting the relative calibration accuracy}

A closer look at Figure 3 reveals obvious trends in all three bands. There is a long time trend showing that the flux ratios (thus the measured fluxes) are getting smaller as time progresses. Also there is a variation on shorter timescale. The intrinsic accuracy of the photometry and the repeatability of the measurements can be increased to large extent by taking into account two issues that are mainly responsible for these variations and affect the flux measured on the array.

\section{$6.1{ }^{3} \mathrm{He}$ cooler evaporator temperature}

[11] showed a good correlation between the temperature of the evaporator and the differential calibration block signal (see Fig. 5. of [11]). Based on this relation a pixel-based correction has been established [11]. Applying this correction to the data after calibrating the responsivity, results in more accurate flux measurements. This correction is typically below $1 \%$. Only $1.2 \%$ of all observations need larger correction. See [11] for details. The task that performs this correction will be available in HIPE 12.0. Figure 5 shows the same as Figure 3 after applying the correction for the evaporator temperature. The scatter of the individual objects has been greatly reduced that leads to the clear recognition of the second effect: the change of the bolometer response with the telescope flux. 

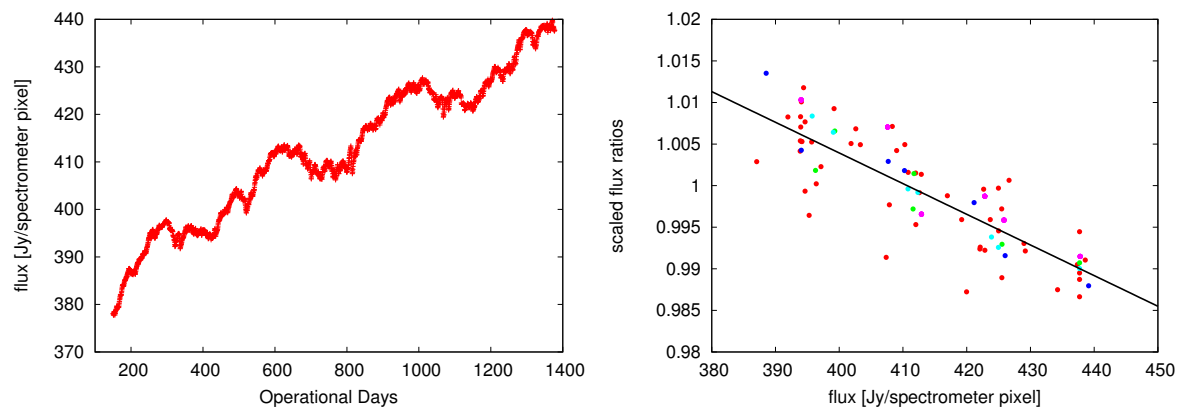

Fig. 6 The $70 \mu \mathrm{m}$ background flux of the main mirror integrated over the beam of a PACS spectrometer pixel $\left(9.4^{\prime \prime} \times 9.4^{\prime \prime}\right)$ as a function of the Operational Days (left panel) and the correlation between the flux ratios of the fiducial stars and the main mirror flux at $70 \mu \mathrm{m}$ (right panel)

\subsection{Variation of telescope background}

It is a general feature of the bolometers that their responsivity decreases with increasing optical load. Examining the photometric variation of $\gamma$ Dra that was monitored regularly during the mission lead to the realization that the flux showed seasonal changes that correlated well with the calculated telescope flux based on the temperature of the main mirror. It is known that the emission of the main mirror is the largest contributor of the flux impinging on the detector (Figure 6 left panel as an example of the $70 \mu \mathrm{m}$ flux (Albrecht Poglitsch private communication)) . This calculation was originally provided for the purpose of Telescope Background Normalization of the spectrometer, therefore the unit is $J y /$ (spectrometer pixel)). The curve on Figure 6 (left panel) can be interpreted as annual temperature variations of the primary, where the bumps occur at the orbit perigee, on top of a slowly increasing emissivity of the primary mirror due to e.g. dust deposition on the reflecting surface, surface degradation by cosmic rays. We can derive a linear correlation between the measured flux ratios and the telescope flux (Figure 6right panel.)

The derived functions are the following:

$$
\begin{array}{r}
f_{\text {blue }}(x)=-0.000369 * x+1.151418 \\
f_{\text {green }}(x)=-0.000884 * x+1.267293 \\
f_{\text {red }}(x)=-0.002811 * x+1.561422
\end{array}
$$

where $x$ is the telescope flux in the appropriate band. Note that to derive these correlations we need to scale the flux ratios of the fiducial stars to bring them on the same level. The scaling would not influence our results because it does not change the slope of the curves. Since we are interested only in the relative change, we divide the measured flux by $f(x) / f(c)$ where $c$ is the flux value where the conversion results in no change $(f(x) / f(c)=1)$. The values of $c$ are 410.65, 302.24 and $199.75 \mathrm{Jy} /$ (spectrometer pixel) in the blue, green and red band respectively. The maximum effect of this correction is around $1.5 \%$.

\section{Finally achievable calibration accuracies}

The result of the calibrations after applying both the evaporator temperature correction and the telescope flux correction are summarised in Table 4 
Table 4 Observed and calibrated ("FM, 7") monochromatic flux densities at 70, 100, $160 \mu \mathrm{m}$ divided by the corresponding current model predictions for all 5 fiducial stars after the evaporator temperature and the telescope flux corrections.

\begin{tabular}{lrrrrrrrrr}
\hline \multirow{2}{*}{$\begin{array}{l}\text { Target } \\
\text { name }\end{array}$} & \multicolumn{3}{c}{ blue obs/model } & \multicolumn{3}{c}{ green obs/model } & \multicolumn{3}{c}{ red obs/model } \\
& no. & mean & stdev & no. & mean & stdev & no. & mean & stdev \\
\hline$\beta$ And & 6 & $\mathbf{1 . 0 2 5}$ & 0.003 & 6 & $\mathbf{1 . 0 2 4}$ & 0.004 & 6 & $\mathbf{0 . 9 9 7}$ & 0.008 \\
$\alpha$ Cet & 6 & $\mathbf{1 . 0 1 3}$ & 0.006 & 6 & $\mathbf{1 . 0 1 9}$ & 0.003 & 6 & $\mathbf{1 . 0 2 8}$ & 0.016 \\
$\alpha$ Tau & 5 & $\mathbf{0 . 9 8 1}$ & 0.002 & 5 & $\mathbf{0 . 9 8 5}$ & 0.003 & 5 & $\mathbf{0 . 9 8 0}$ & 0.009 \\
$\alpha$ Boo & 7 & $\mathbf{0 . 9 9 6}$ & 0.003 & 7 & $\mathbf{1 . 0 0 3}$ & 0.002 & 7 & $\mathbf{1 . 0 0 9}$ & 0.011 \\
$\gamma$ Dra & 49 & $\mathbf{0 . 9 9 1}$ & 0.004 & 10 & $\mathbf{0 . 9 9 4}$ & 0.006 & 54 & $\mathbf{1 . 0 1 5}$ & 0.027 \\
\hline \multicolumn{2}{l}{ mean/stdev } & $\mathbf{1 . 0 0 3} \pm \mathbf{0 . 0 1 9}$ & & $\mathbf{1 . 0 0 5} \pm \mathbf{0 . 0 1 7}$ & & $\mathbf{1 . 0 0 6} \pm \mathbf{0 . 0 1 8}$ \\
\hline
\end{tabular}
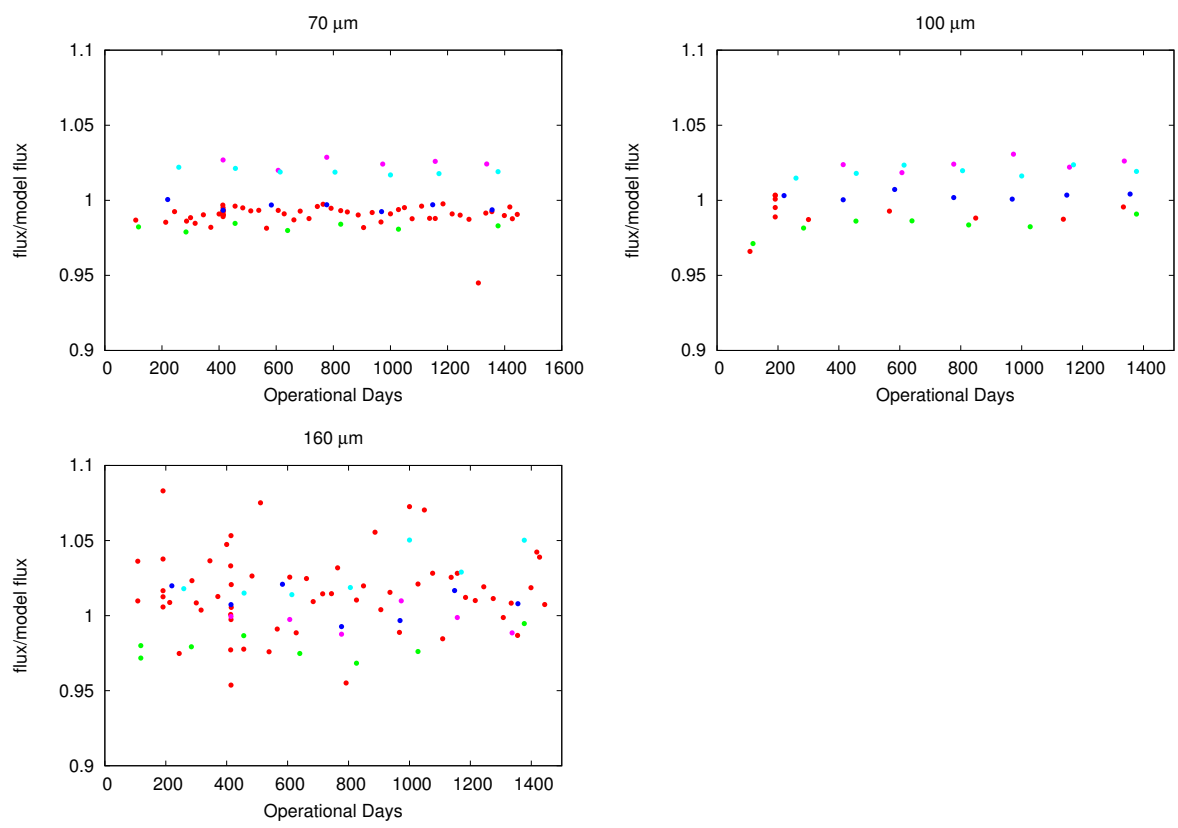

Fig. 7 Measured over model flux ratios of the five fiducial standards at $70 \mu \mathrm{m}$ (upper left), $100 \mu \mathrm{m}$ (upper right) and $160 \mu \mathrm{m}$ (lower left) after applying the corrections for the evaporator temperature and the main mirror flux.

Although the overall accuracy of the calibration did not improve significantly, the uncertainties of the individual sources were reduced at least by a factor of two in the blue band and 1.5 in the green band to bring the intrinsic accuracy (repeatability) of the individual calibration sources to around $0.5 \%$. In the red band the corrections do not improve the scatter of the individual sources significantly. Here the conservative estimate of the repeatability remained around $2 \%$.

Figure 7 shows again the same as Figure 3 and 5 after applying the correction for the evaporator temperature and the telescope flux. The trend with time observable especially in the blue band (left panel) in Figure 5 disappears. 


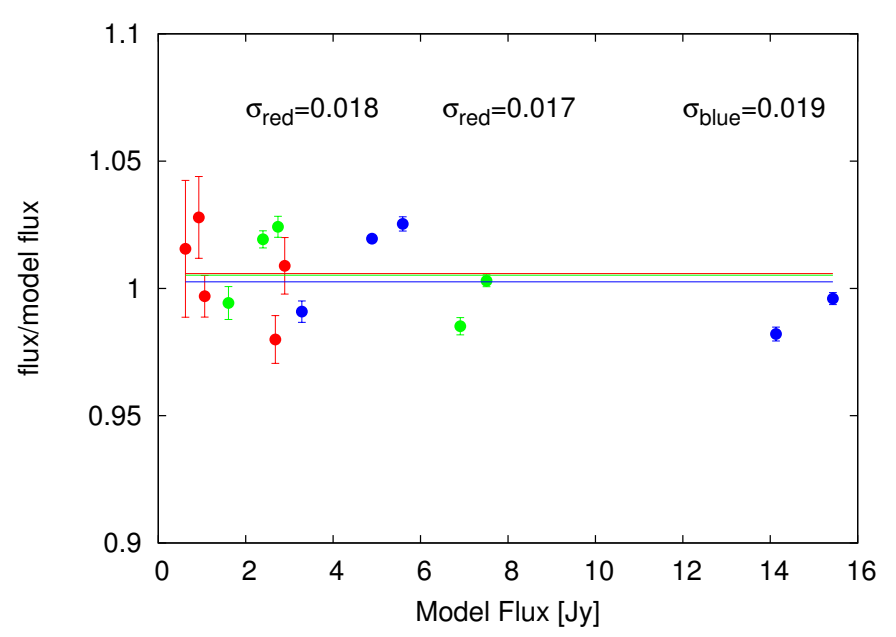

Fig. 8 Final result after applying the corrections. Red dots: $160 \mu \mathrm{m}$; green dots: $100 \mu \mathrm{m}$; blue dots: $70 \mu \mathrm{m}$. The red, green and blue lines represent the average values at $160 \mu \mathrm{m}, 100 \mu \mathrm{m}$, and $70 \mu \mathrm{m}$ respectively.

Finally, in Figure 8 we reproduce Figure 4 using the corrected photometry. Note the decrease of the size of the error bars in the blue and green band in Figure 8 with respect to Figure 4

\section{Conclusions}

We presented a quality check of the flux calibration scheme of the PACS photometer implemented in HIPE. Using the latest available responsivity calibration file (FM 7) we found that the measured fluxes of the five fiducial stars are in very good agreement with the model fluxes confirming our calibration and data reduction procedures. However we realize that there is a clear discrepancy (3-4\%) between the calibrators with different spectral types. The intrinsic uncertainty of the photometry of the individual sources is around or slightly below $2 \%$. Adding the total of $5 \%$ uncertainty of the stellar models gives $7 \%$ as the most conservative estimate for the final error of our absolute flux calibration. Therefore the overall uncertainty of the calibration is dominated by the models. We do not see any systematic wavelength dependence in the calibration. The intrinsic accuracy of the photometry is affected by two instrumental effects. We have shown that these effects can be calibrated out in the blue and green bands bringing the intrinsic accuracy in these bands down to around $0.5 \%$. It is important to note that these result are closely related to the way the observations were reduced. Different settings in the reduction scripts (for the masks, the deglitching, the high-pass filter widths, etc.) might produce slightly different fluxes. If one wants to be sure that the calibration is $100 \%$ correct, then one has to take all OBSIDs of the 5 fiducial stars as listed in Tables 5, 6, 7, 8, and 9 and re-process the data with his/her own reduction script. Based on the new flux/model ratios one would have to adjust the fluxes of the science observations of interest. These adjustments are expected to be on a few percent level. 
Acknowledgements We would like to thank the anonymous referee for the comments and suggestions that significantly improved the manuscript. Z. Balog, H. Linz and M. Nielbock are funded by the Deutsches Zentrum für Luft- und Raumfahrt e. V.

\section{References}

1. Absil O. et al., Circumstellar material in the Vega inner system revealed by CHARA/FLUOR, A\&A, 452, 237 (2006)

2. Billot N. et al., The Herschel-PACS 2560 bolometers imaging camera, SPIE Proc., 6265, article id. 62650D, 12 pp. (2006)

3. Cohen M. et al., Stellar calibration in the infrared: extending the lagacy of the KAO, ISO, and MSX to SIRTF and beyond, Proceedings of the Conference "The Calibration Legacy of the ISO Mission", ESA SP-481, 135 (2003)

4. Decin L. et al. ISO-SWS calibration and the accurate modelling of cool-star atmospheres: IV. G9 to M2 stars, A\&A, 400, 709 (2003)

5. Decin L. \& Eriksson K., Theoretical model atmosphere spectra used for the calibration of infrared instruments, A\&A, 472, 1041 (2007)

6. Dehaes S. et al., Structure of the outer layers of cool standard stars, A\&A, 533, 107 (2011)

7. Ducati J.R., Catalogue of Stellar Photometry in Johnson's 11-color system., CDS/ADC Collection of Electronic Catalogues, 2237, 0 (2002)

8. Gordon K. et al., Absolute calibration and characterization of the multiband imaging photometer for Spitzer. II. $70 \mu \mathrm{m}$ imaging, PASP, 119, 1019 (2007)

9. Gustafsson B. et al., A grid of model atmospheres for metal-deficient giant stars. I, A\&A, 42, 407 (1975)

10. Gustafsson B. et al., A Grid of Model Atmospheres for Cool Stars. Proceedings of the Conference "Stellar Atmosphere Modeling", ASP Conference Series 288, 331 (2003)

11. Moór et al., PACS photometer calibration block analysis, Experimental Astronomy, this volume (2013)

12. Moreno R., Neptune and Uranus planetry brightness temperature tabulation, Tech. rep., ESA Herschel Science Centre, available from ftp://ftp.sciops.esa.int/pub/hsc-calibration/PlanetrayModels/ESA4/(2012)

13. Müller Th. et al., Asteroid calibration, Experimental Astronomy, this volume (2013)

14. Nielbock M. et al., The Herschel-PACS photometer calibration; A time dependent flux calibration for the PACS chopped photometry AOT mode, Experimental Astronomy, this volume (2013)

15. Pilbratt G. et al., Herschel Space Observatory, A\&A, 518, L1 (2010)

16. Plez B. et al., Spherical opacity sampling model atmospheres for M-giants. I - Techniques, data and discussion A\&A, 256, 551 (1992)

17. Poglitsch A. et al., The Photodetector Array Camera and Spectrometer (PACS) on the Herschel Space Observatory, A\&A, 518, L2 (2010)

18. Popesso, P. et al., The effect of the high-pass filter data reduction technique on the Herschel PACS Photometer PSF and noise, submitted to A\&A, arXiv:1211.4257(2013)

19. Price S.D. et al., Spectral Irradiance Calibration in the Infrared. XV. Absolute Calibration of Standard Stars by Experiments on the Midcourse Space Experiment, AJ, 128, 889 (2004)

20. Rieke G. et al., An absolute photometric system at 10 and 20 microns, AJ, 90, 900 (1985)

21. Selby M.J. et al., Narrow band 1 micron-4 micron infrared photometry of 176 stars, A\&AS, 74, 127 (1988) 
Appendix

Table 5 PACS photometer observation details for $\alpha$ Boo (HR 5340; HD 124897; HIP 87833; Arcturus).

\begin{tabular}{llccrrrr}
\hline \multirow{2}{*}{ OD } & OBSIDs & $\begin{array}{c}\text { filter } \\
\text { bands }\end{array}$ & $\begin{array}{c}\text { scan-angles } \\
{[\mathrm{deg}]}\end{array}$ & $\begin{array}{c}\text { scan-legs } \\
\text { len ['] }\end{array}$ & $\begin{array}{l}\text { notes/ } \\
\text { sep ["] }\end{array}$ & no. & remarks \\
\hline \multirow{2}{*}{220} & 1342188245,1342188246 & $\mathrm{~b} / \mathrm{r}$ & $63 / 117$ & 4.0 & 4.0 & 8 & \\
& 1342188247,1342188248 & $\mathrm{~g} / \mathrm{r}$ & $63 / 117$ & 4.0 & 4.0 & 8 & \\
\multirow{2}{*}{414} & 1342199603,1342199604 & $\mathrm{~b} / \mathrm{r}$ & $70 / 110$ & 3.0 & 4.0 & 10 & \\
& 1342199606,1342199607 & $\mathrm{~g} / \mathrm{r}$ & $70 / 110$ & 3.0 & 4.0 & 10 & \\
583 & 1342211280,1342211281 & $\mathrm{~b} / \mathrm{r}$ & $70 / 110$ & 3.0 & 4.0 & 10 & \\
& 1342211283,1342211284 & $\mathrm{~g} / \mathrm{r}$ & $70 / 110$ & 3.0 & 4.0 & 10 & \\
777 & 1342223345,1342223346 & $\mathrm{~b} / \mathrm{r}$ & $70 / 110$ & 3.0 & 4.0 & 10 & \\
& 1342223348,1342223349 & $\mathrm{~g} / \mathrm{r}$ & $70 / 110$ & 3.0 & 4.0 & 10 & \\
\multirow{2}{*}{969} & 1342236968,1342236969 & $\mathrm{~b} / \mathrm{r}$ & $70 / 110$ & 3.0 & 4.0 & 10 & \\
& 1342236965,1342236966 & $\mathrm{~g} / \mathrm{r}$ & $70 / 110$ & 3.0 & 4.0 & 10 & \\
\multirow{2}{*}{1148} & 1342247705,1342247706 & $\mathrm{~b} / \mathrm{r}$ & $70 / 110$ & 3.0 & 4.0 & 10 & \\
& 1342247702,1342247703 & $\mathrm{~g} / \mathrm{r}$ & $70 / 110$ & 3.0 & 4.0 & 10 & \\
& 1342262519,1342262520 & $\mathrm{~b} / \mathrm{r}$ & $70 / 110$ & 3.0 & 4.0 & 10 & \\
& 1342262516,1342262517 & $\mathrm{~g} / \mathrm{r}$ & $70 / 110$ & 3.0 & 4.0 & 10 & \\
\hline
\end{tabular}

Table 6 PACS photometer observation details for $\alpha$ Cet (HR 911; HD 18884; HIP 14135; Menkar).

\begin{tabular}{|c|c|c|c|c|c|c|c|}
\hline OD & OBSIDs & $\begin{array}{c}\text { filter } \\
\text { bands }\end{array}$ & $\begin{array}{c}\text { scan-angles } \\
\text { [deg] }\end{array}$ & len $\left[{ }^{\prime}\right]$ & $\begin{array}{l}\text { scan-legs } \\
\text { sep ["] }\end{array}$ & no & $\begin{array}{l}\text { notes/ } \\
\text { remarks }\end{array}$ \\
\hline \multirow[t]{2}{*}{259} & 1342189824,1342189825 & $\mathrm{~b} / \mathrm{r}$ & $63 / 117$ & 4.0 & 4.0 & 8 & \\
\hline & 1342189827,1342189828 & $\mathrm{~g} / \mathrm{r}$ & $63 / 117$ & 4.0 & 4.0 & 8 & \\
\hline \multirow[t]{2}{*}{457} & 1342203030,1342203031 & $\mathrm{~b} / \mathrm{r}$ & $70 / 110$ & 3.0 & 4.0 & 10 & \\
\hline & 1342203033,1342203034 & $\mathrm{~g} / \mathrm{r}$ & $70 / 110$ & 3.0 & 4.0 & 10 & \\
\hline \multirow[t]{2}{*}{614} & 1342212856,1342212857 & $\mathrm{~b} / \mathrm{r}$ & $70 / 110$ & 3.0 & 4.0 & 10 & \\
\hline & 1342212853,1342212854 & $\mathrm{~g} / \mathrm{r}$ & $70 / 110$ & 3.0 & 4.0 & 10 & \\
\hline \multirow[t]{2}{*}{806} & 1342224927,1342224928 & $\mathrm{~b} / \mathrm{r}$ & $70 / 110$ & 3.0 & 4.0 & 10 & \\
\hline & 1342224930,1342224931 & $\mathrm{~g} / \mathrm{r}$ & $70 / 110$ & 3.0 & 4.0 & 10 & \\
\hline \multirow[t]{2}{*}{1000} & 1342238782,1342238783 & $\mathrm{~b} / \mathrm{r}$ & $70 / 110$ & 3.0 & 4.0 & 10 & \\
\hline & 1342238779,1342238780 & $\mathrm{~g} / \mathrm{r}$ & $70 / 110$ & 3.0 & 4.0 & 10 & \\
\hline \multirow[t]{2}{*}{1170} & 1342248722,1342248723 & $\mathrm{~b} / \mathrm{r}$ & $70 / 110$ & 3.0 & 4.0 & 10 & \\
\hline & 1342248719,1342248720 & $\mathrm{~g} / \mathrm{r}$ & $70 / 110$ & 3.0 & 4.0 & 10 & \\
\hline \multirow[t]{2}{*}{1377} & 1342263910,1342263911 & $\mathrm{~b} / \mathrm{r}$ & $70 / 110$ & 3.0 & 4.0 & 10 & \\
\hline & 1342263907,1342263908 & $\mathrm{~g} / \mathrm{r}$ & $70 / 110$ & 3.0 & 4.0 & 10 & \\
\hline
\end{tabular}


Table 7 PACS photometer observation details for $\alpha$ Tau (HR 1457; HD 29139; HIP 21421; Aldebaran).

\begin{tabular}{|c|c|c|c|c|c|c|c|}
\hline OD & OBSIDs & $\begin{array}{l}\text { filter } \\
\text { bands }\end{array}$ & $\begin{array}{c}\text { scan-angles } \\
\text { [deg] }\end{array}$ & len $\left[^{\prime}\right]$ & $\begin{array}{l}\text { scan-legs } \\
\left.\text { sep [" }{ }^{\prime \prime}\right]\end{array}$ & no & $\begin{array}{l}\text { notes/ } \\
\text { remarks }\end{array}$ \\
\hline \multirow[t]{2}{*}{118} & 1342183532,1342183533 & $\mathrm{~b} / \mathrm{r}$ & $45 / 135$ & 5.0 & 51.0 & 4 & very low coverage \\
\hline & 1342183534,1342183535 & $\mathrm{~g} / \mathrm{r}$ & $45 / 135$ & 5.0 & 51.0 & 4 & very low coverage \\
\hline \multirow[t]{2}{*}{118} & 1342183538 & $\mathrm{~b} / \mathrm{r}$ & 63 & 10.0 & 3.0 & 15 & no cross-scan \\
\hline & 1342183541 & $\mathrm{~g} / \mathrm{r}$ & 63 & 10.0 & 3.0 & 15 & no cross-scan \\
\hline \multirow[t]{2}{*}{284} & 1342190947,1342190948 & $\mathrm{~b} / \mathrm{r}$ & $70 / 110$ & 2.5 & 4.0 & 10 & \\
\hline & 1342190944,1342190945 & $\mathrm{~g} / \mathrm{r}$ & $70 / 110$ & 2.5 & 4.0 & 10 & \\
\hline \multirow[t]{2}{*}{456} & 1342202961,1342202962 & $\mathrm{~b} / \mathrm{r}$ & $70 / 110$ & 2.5 & 4.0 & 10 & \\
\hline & 1342202958,1342202959 & $\mathrm{~g} / \mathrm{r}$ & $70 / 110$ & 2.5 & 4.0 & 10 & \\
\hline \multirow[t]{2}{*}{640} & 1342214211,1342214212 & $\mathrm{~b} / \mathrm{r}$ & $70 / 110$ & 3.0 & 4.0 & 10 & \\
\hline & 1342214214,1342214215 & $\mathrm{~g} / \mathrm{r}$ & $70 / 110$ & 3.0 & 4.0 & 10 & \\
\hline \multirow[t]{2}{*}{826} & 1342226740,1342226741 & $\mathrm{~b} / \mathrm{r}$ & $70 / 110$ & 3.0 & 4.0 & 10 & \\
\hline & 1342226743,1342226744 & $\mathrm{~g} / \mathrm{r}$ & $70 / 110$ & 3.0 & 4.0 & 10 & \\
\hline \multirow[t]{2}{*}{1028} & 1342240755,1342240756 & $\mathrm{~b} / \mathrm{r}$ & $70 / 110$ & 3.0 & 4.0 & 10 & \\
\hline & 1342240758,1342240759 & $\mathrm{~g} / \mathrm{r}$ & $70 / 110$ & 3.0 & 4.0 & 10 & \\
\hline \multirow[t]{2}{*}{1377} & 1342263918,1342263919 & $\mathrm{~b} / \mathrm{r}$ & $70 / 110$ & 3.0 & 4.0 & 10 & \\
\hline & 1342263915,1342263916 & $\mathrm{~g} / \mathrm{r}$ & $70 / 110$ & 3.0 & 4.0 & 10 & \\
\hline
\end{tabular}

Table 8 PACS photometer observation details for $\beta$ And (HR 337; HD 6860; HIP 5447; Mirach).

\begin{tabular}{|c|c|c|c|c|c|c|c|}
\hline OD & OBSIDs & $\begin{array}{c}\text { filter } \\
\text { bands }\end{array}$ & $\begin{array}{c}\text { scan-angles } \\
\text { [deg] }\end{array}$ & len $\left[{ }^{\prime}\right]$ & $\begin{array}{l}\text { scan-legs } \\
\text { sep [ ["] }\end{array}$ & no & $\begin{array}{l}\text { notes/ } \\
\text { remarks }\end{array}$ \\
\hline \multirow[t]{2}{*}{414} & 1342199609,1342199610 & $\mathrm{~b} / \mathrm{r}$ & $70 / 110$ & 3.0 & 4.0 & 10 & \\
\hline & 1342199612,1342199613 & $\mathrm{~g} / \mathrm{r}$ & $70 / 110$ & 3.0 & 4.0 & 10 & \\
\hline \multirow[t]{2}{*}{607} & 1342212507,1342212508 & $\mathrm{~b} / \mathrm{r}$ & $70 / 110$ & 3.0 & 4.0 & 10 & \\
\hline & 1342212504,1342212505 & $\mathrm{~g} / \mathrm{r}$ & $70 / 110$ & 3.0 & 4.0 & 10 & \\
\hline \multirow[t]{2}{*}{777} & 1342223335,1342223336 & $\mathrm{~b} / \mathrm{r}$ & $70 / 110$ & 3.0 & 4.0 & 10 & \\
\hline & 1342223338,1342223339 & $\mathrm{~g} / \mathrm{r}$ & $70 / 110$ & 3.0 & 4.0 & 10 & \\
\hline \multirow[t]{2}{*}{973} & 1342237164,1342237165 & $\mathrm{~b} / \mathrm{r}$ & $70 / 110$ & 3.0 & 4.0 & 10 & \\
\hline & 1342237161,1342237162 & $\mathrm{~g} / \mathrm{r}$ & $70 / 110$ & 3.0 & 4.0 & 10 & \\
\hline \multirow[t]{2}{*}{1157} & 1342248035,1342248036 & $\mathrm{~b} / \mathrm{r}$ & $70 / 110$ & 3.0 & 4.0 & 10 & \\
\hline & 1342248032,1342248033 & $\mathrm{~g} / \mathrm{r}$ & $70 / 110$ & 3.0 & 4.0 & 10 & \\
\hline \multirow[t]{2}{*}{1337} & 1342259260,1342259261 & $\mathrm{~b} / \mathrm{r}$ & $70 / 110$ & 3.0 & 4.0 & 10 & \\
\hline & 1342259257,1342259258 & $\mathrm{~g} / \mathrm{r}$ & $70 / 110$ & 3.0 & 4.0 & 10 & \\
\hline
\end{tabular}


Table 9 PACS photometer observation details for $\gamma$ Dra (HR 6705; HD 164058; HIP 87833; Etamin).

\begin{tabular}{|c|c|c|c|c|c|c|c|}
\hline OD & OBSIDs & $\begin{array}{c}\text { filter } \\
\text { bands }\end{array}$ & $\begin{array}{c}\text { scan-angles } \\
\text { [deg] }\end{array}$ & len $\left[^{\prime}\right]$ & $\begin{array}{l}\text { scan-legs } \\
\left.\text { sep [" }{ }^{\prime \prime}\right]\end{array}$ & no & $\begin{array}{l}\text { notes/ } \\
\text { remarks }\end{array}$ \\
\hline 108 & 1342182985,1342182987 & $\mathrm{~b} / \mathrm{r}$ & $45 / 135$ & 5.0 & 51.0 & 4 & very low coverage \\
\hline 108 & 1342182997,1342182980 & $\mathrm{~g} / \mathrm{r}$ & $45 / 135$ & 5.0 & 51.0 & 4 & very low coverage \\
\hline \multirow[t]{2}{*}{108} & 1342182986 & $\mathrm{~b} / \mathrm{r}$ & 45 & 30.0 & 4.0 & 15 & no cross-scan \\
\hline & 1342182981 & $\mathrm{~g} / \mathrm{r}$ & 45 & 30.0 & 4.0 & 15 & no cross-scan \\
\hline \multirow[t]{5}{*}{191} & 1342187147,1342187148 & $\mathrm{~g} / \mathrm{r}$ & $63 / 117$ & 3.9 & 5.0 & 8 & \\
\hline & 1342187149,1342187150 & $\mathrm{~g} / \mathrm{r}$ & $63 / 117$ & 3.9 & 4.0 & 8 & \\
\hline & 1342187151,1342187152 & $\mathrm{~g} / \mathrm{r}$ & $63 / 117$ & 3.0 & 4.0 & 8 & \\
\hline & 1342187153,1342187154 & $\mathrm{~g} / \mathrm{r}$ & $63 / 117$ & 3.9 & 2.0 & 16 & \\
\hline & 1342187155,1342187156 & $\mathrm{~g} / \mathrm{r}$ & $63 / 117$ & 3.0 & 2.0 & 16 & \\
\hline 213 & 1342188070,1342188071 & $\mathrm{~b} / \mathrm{r}$ & $63 / 117$ & 4.0 & 4.0 & 8 & \\
\hline 244 & 1342189187,1342189187 & $\mathrm{~b} / \mathrm{r}$ & $63 / 117$ & 4.0 & 4.0 & 8 & \\
\hline 286 & 1342191125,1342191126 & $\mathrm{~b} / \mathrm{r}$ & $70 / 110$ & 2.5 & 4.0 & 10 & \\
\hline \multirow[t]{2}{*}{300} & 1342191958,1342191959 & $\mathrm{~b} / \mathrm{r}$ & $70 / 110$ & 2.5 & 4.0 & 10 & \\
\hline & 1342191961,1342191962 & $\mathrm{~g} / \mathrm{r}$ & $70 / 110$ & 2.5 & 4.0 & 10 & \\
\hline 316 & 1342192780,1342192781 & $\mathrm{~b} / \mathrm{r}$ & $70 / 110$ & 2.5 & 4.0 & 10 & \\
\hline 345 & 1342195483,1342195484 & $\mathrm{~b} / \mathrm{r}$ & $70 / 110$ & 2.5 & 4.0 & 10 & \\
\hline 371 & 1342196730,1342196731 & $\mathrm{~b} / \mathrm{r}$ & $70 / 110$ & 2.5 & 4.0 & 10 & \\
\hline 400 & 1342198499,1342198500 & $\mathrm{~b} / \mathrm{r}$ & $70 / 110$ & 3.0 & 4.0 & 10 & \\
\hline \multirow[t]{3}{*}{413} & 1342199481,1342199482 & $\mathrm{~b} / \mathrm{r}$ & $70 / 110$ & 3.0 & 4.0 & 10 & \\
\hline & 1342199512,1342199513 & $\mathrm{~b} / \mathrm{r}$ & $70 / 110$ & 3.0 & 4.0 & 10 & \\
\hline & 1342199526,1342199527 & $\mathrm{~b} / \mathrm{r}$ & $70 / 110$ & 3.0 & 4.0 & 10 & \\
\hline \multirow[t]{3}{*}{414} & 1342199600,1342199601 & $\mathrm{~b} / \mathrm{r}$ & $70 / 110$ & 3.0 & 4.0 & 10 & \\
\hline & 1342199639,1342199640 & $\mathrm{~b} / \mathrm{r}$ & $70 / 110$ & 3.0 & 4.0 & 10 & \\
\hline & 1342199655,1342199656 & $\mathrm{~b} / \mathrm{r}$ & $70 / 110$ & 3.0 & 4.0 & 10 & \\
\hline \multirow[t]{2}{*}{415} & 1342199707,1342199708 & $\mathrm{~b} / \mathrm{r}$ & $70 / 110$ & 3.0 & 4.0 & 10 & \\
\hline & 1342199717,1342199718 & $\mathrm{~b} / \mathrm{r}$ & $70 / 110$ & 3.0 & 4.0 & 10 & \\
\hline 456 & 1342202942,1342202943 & $\mathrm{~b} / \mathrm{r}$ & $70 / 110$ & 3.0 & 4.0 & 10 & \\
\hline 483 & 1342204209,1342204210 & $\mathrm{~b} / \mathrm{r}$ & $70 / 110$ & 3.0 & 4.0 & 10 & \\
\hline 511 & 1342206001,1342206002 & $\mathrm{~b} / \mathrm{r}$ & $70 / 110$ & 3.0 & 4.0 & 10 & \\
\hline 539 & 1342208971,1342208972 & $\mathrm{~b} / \mathrm{r}$ & $70 / 110$ & 3.0 & 4.0 & 10 & \\
\hline \multirow[t]{2}{*}{566} & 1342210582,1342210583 & $\mathrm{~b} / \mathrm{r}$ & $70 / 110$ & 3.0 & 4.0 & 10 & \\
\hline & 1342210584,1342210585 & $\mathrm{~g} / \mathrm{r}$ & $70 / 110$ & 3.0 & 4.0 & 10 & \\
\hline 607 & 1342212494,1342212495 & $\mathrm{~b} / \mathrm{r}$ & $70 / 110$ & 3.0 & 4.0 & 10 & \\
\hline 628 & 1342213588,1342213589 & $\mathrm{~b} / \mathrm{r}$ & $70 / 110$ & 3.0 & 4.0 & 10 & \\
\hline 662 & 1342215374,1342215375 & $\mathrm{~b} / \mathrm{r}$ & $70 / 110$ & 3.0 & 4.0 & 10 & \\
\hline 684 & 1342217404,1342217405 & $\mathrm{~b} / \mathrm{r}$ & $70 / 110$ & 3.0 & 4.0 & 10 & \\
\hline 715 & 1342220823,1342220824 & $\mathrm{~b} / \mathrm{r}$ & $70 / 110$ & 3.0 & 4.0 & 10 & \\
\hline 744 & 1342221811,1342221812 & $\mathrm{~b} / \mathrm{r}$ & $70 / 110$ & 3.0 & 4.0 & 10 & \\
\hline 764 & 1342222756,1342222757 & $\mathrm{~b} / \mathrm{r}$ & $70 / 110$ & 3.0 & 4.0 & 10 & \\
\hline 792 & 1342224229,1342224230 & $\mathrm{~b} / \mathrm{r}$ & $70 / 110$ & 3.0 & 4.0 & 10 & \\
\hline 826 & 1342226712,1342226713 & $\mathrm{~b} / \mathrm{r}$ & $70 / 110$ & 3.0 & 4.0 & 10 & \\
\hline \multirow[t]{2}{*}{849} & 1342228388,1342228389 & $\mathrm{~b} / \mathrm{r}$ & $70 / 110$ & 3.0 & 4.0 & 10 & \\
\hline & 1342228391,1342228392 & $\mathrm{~g} / \mathrm{r}$ & $70 / 110$ & 3.0 & 4.0 & 10 & \\
\hline 887 & 1342231097,1342231098 & $\mathrm{~b} / \mathrm{r}$ & $70 / 110$ & 3.0 & 4.0 & 10 & \\
\hline 906 & 1342231899,1342231900 & $\mathrm{~b} / \mathrm{r}$ & $70 / 110$ & 3.0 & 4.0 & 10 & \\
\hline 936 & 1342234214,1342234215 & $\mathrm{~b} / \mathrm{r}$ & $70 / 110$ & 3.0 & 4.0 & 10 & \\
\hline 967 & 1342237975,1342237976 & $\mathrm{~b} / \mathrm{r}$ & $70 / 110$ & 3.0 & 4.0 & 10 & \\
\hline 1000 & 1342238772,1342238773 & $\mathrm{~b} / \mathrm{r}$ & $70 / 110$ & 3.0 & 4.0 & 10 & \\
\hline 1028 & 1342240699,1342240700 & $\mathrm{~b} / \mathrm{r}$ & $70 / 110$ & 3.0 & 4.0 & 10 & \\
\hline 1049 & 1342242557,1342242558 & $\mathrm{~b} / \mathrm{r}$ & $70 / 110$ & 3.0 & 4.0 & 10 & \\
\hline 1076 & 1342244900,1342244901 & $\mathrm{~b} / \mathrm{r}$ & $70 / 110$ & 3.0 & 4.0 & 10 & \\
\hline 1109 & 1342246181,1342246182 & $\mathrm{~b} / \mathrm{r}$ & $70 / 110$ & 3.0 & 4.0 & 10 & \\
\hline \multirow[t]{2}{*}{1137} & 1342247335,1342247336 & $\mathrm{~b} / \mathrm{r}$ & $70 / 110$ & 3.0 & 4.0 & 10 & \\
\hline & 1342247338,1342247339 & $\mathrm{~g} / \mathrm{r}$ & $70 / 110$ & 3.0 & 4.0 & 10 & \\
\hline 1157 & 1342248038,1342248039 & $\mathrm{~b} / \mathrm{r}$ & $70 / 110$ & 3.0 & 4.0 & 10 & \\
\hline 1184 & 1342249293,1342249294 & $\mathrm{~b} / \mathrm{r}$ & $70 / 110$ & 3.0 & 4.0 & 10 & \\
\hline 1216 & 1342250856,1342250857 & $\mathrm{~b} / \mathrm{r}$ & $70 / 110$ & 3.0 & 4.0 & 10 & \\
\hline 1244 & 1342252805,1342252806 & $\mathrm{~b} / \mathrm{r}$ & $70 / 110$ & 3.0 & 4.0 & 10 & \\
\hline 1275 & 1342254723,1342254724 & $\mathrm{~b} / \mathrm{r}$ & $70 / 110$ & 3.0 & 4.0 & 10 & \\
\hline 1308 & 1342256959,1342256960 & $\mathrm{~b} / \mathrm{r}$ & $70 / 110$ & 3.0 & 4.0 & 10 & \\
\hline \multirow[t]{2}{*}{1334} & 1342258831,1342258832 & $\mathrm{~b} / \mathrm{r}$ & $70 / 110$ & 3.0 & 4.0 & 10 & \\
\hline & 1342258834,1342258835 & $\mathrm{~g} / \mathrm{r}$ & $70 / 110$ & 3.0 & 4.0 & 10 & \\
\hline 1355 & 1342262225,1342262226 & $\mathrm{~b} / \mathrm{r}$ & $70 / 110$ & 3.0 & 4.0 & 10 & \\
\hline 1399 & 1342267291,1342267292 & $\mathrm{~b} / \mathrm{r}$ & $70 / 110$ & 3.0 & 4.0 & 10 & \\
\hline 1418 & 1342268966,1342268967 & $\mathrm{~b} / \mathrm{r}$ & $70 / 110$ & 3.0 & 4.0 & 10 & \\
\hline 1427 & 1342269812,1342269813 & $\mathrm{~b} / \mathrm{r}$ & $70 / 110$ & 3.0 & 4.0 & 10 & \\
\hline 1444 & 1342271000,1342271001 & $\mathrm{~b} / \mathrm{r}$ & $70 / 110$ & 3.0 & 4.0 & 10 & \\
\hline
\end{tabular}


Table 10 PACS photometery of $\alpha$ Tau. "with EVC" - fluxes taking into account the correction for the evaporator temperature (see Section 6.1); "without EVC" - fluxes without taking into account the correction for the evaporator temperature. This is also valid for Table \begin{tabular}{|l|l|l|l|}
\hline 11 & 12 & 13 & 14 \\
\hline
\end{tabular}

\begin{tabular}{|c|c|c|c|c|c|c|c|c|c|c|c|c|}
\hline \multirow[t]{3}{*}{ OD } & \multicolumn{12}{|c|}{ filter } \\
\hline & \multicolumn{4}{|c|}{ blue $(70 \mu \mathrm{m})$} & \multicolumn{4}{|c|}{ green $(100 \mu \mathrm{m})$} & \multicolumn{4}{|c|}{ red $(160 \mu \mathrm{m})$} \\
\hline & \multicolumn{2}{|c|}{ with EVC } & \multicolumn{2}{|c|}{ without EVC } & \multicolumn{2}{|c|}{ with EVC } & \multicolumn{2}{|c|}{ without EVC } & \multicolumn{2}{|c|}{ with EVC } & \multicolumn{2}{|c|}{ without EVC } \\
\hline & flux[Jy] & error[Jy] & flux[Jy] & error[Jy] & flux[Jy] & error[Jy] & flux[Jy] & error[Jy] & flux[Jy] & error[Jy] & flux[Jy] & error[Jy] \\
\hline 118 & 14.281 & 0.011 & 14.281 & 0.011 & 6.997 & 0.022 & 6.993 & 0.022 & 2.818 & 0.035 & 2.817 & 0.035 \\
\hline 284 & 14.128 & 0.006 & 14.128 & 0.006 & 7.020 & 0.006 & 7.020 & 0.006 & 2.817 & 0.027 & 2.817 & 0.027 \\
\hline 456 & 14.122 & 0.007 & 14.122 & 0.007 & 7.019 & 0.002 & 7.054 & 0.002 & 2.828 & 0.031 & 2.841 & 0.031 \\
\hline 640 & 13.858 & 0.005 & 13.858 & 0.005 & 6.907 & 0.010 & 7.030 & 0.010 & 2.758 & 0.029 & 2.797 & 0.029 \\
\hline 826 & 13.642 & 0.003 & 13.642 & 0.003 & 6.740 & 0.007 & 7.020 & 0.007 & 2.695 & 0.037 & 2.786 & 0.039 \\
\hline 1028 & 13.801 & 0.009 & 13.801 & 0.009 & 6.864 & 0.002 & 6.983 & 0.002 & 2.766 & 0.028 & 2.797 & 0.028 \\
\hline 1377 & 13.827 & 0.006 & 13.827 & 0.006 & 6.963 & 0.006 & 7.027 & 0.006 & 2.823 & 0.021 & 2.848 & 0.021 \\
\hline
\end{tabular}

Table 11 PACS photometery of $\alpha$ Boo

\begin{tabular}{|c|c|c|c|c|c|c|c|c|c|c|c|c|}
\hline \multirow[t]{3}{*}{ OD } & \multicolumn{12}{|c|}{ filter } \\
\hline & \multicolumn{4}{|c|}{ blue $(70 \mu \mathrm{m})$} & \multicolumn{4}{|c|}{ green $(100 \mu \mathrm{m})$} & \multicolumn{4}{|c|}{ red $(160 \mu \mathrm{m})$} \\
\hline & \multicolumn{2}{|c|}{ with EVC } & \multicolumn{2}{|c|}{ without EVC } & \multicolumn{2}{|c|}{ with EVC } & \multicolumn{2}{|c|}{ without EVC } & \multicolumn{2}{|c|}{ with EVC } & \multicolumn{2}{|c|}{ without EVC } \\
\hline & flux [Jy] & error[Jy] & flux[Jy] & error[Jy] & flux [Jy] & error[Jy] & flux[Jy] & error[Jy] & flux[Jy] & error [Jy] & flux[Jy] & error[Jy] \\
\hline 220 & 15.839 & 0.007 & 15.817 & 0.007 & 7.828 & 0.009 & 7.818 & 0.009 & 3.181 & 0.044 & 3.177 & 0.044 \\
\hline 414 & 15.593 & 0.013 & 15.672 & 0.013 & 7.752 & 0.010 & 7.791 & 0.010 & 3.125 & 0.031 & 3.138 & 0.031 \\
\hline 583 & 15.655 & 0.004 & 15.634 & 0.004 & 7.812 & 0.008 & 7.803 & 0.007 & 3.167 & 0.058 & 3.163 & 0.058 \\
\hline 777 & 15.197 & 0.010 & 15.651 & 0.011 & 7.521 & 0.006 & 7.781 & 0.006 & 3.003 & 0.028 & 3.088 & 0.029 \\
\hline 969 & 15.302 & 0.001 & 15.475 & 0.001 & 7.652 & 0.009 & 7.726 & 0.009 & 3.053 & 0.050 & 3.082 & 0.050 \\
\hline 1148 & 15.438 & 0.007 & 15.574 & 0.007 & 7.710 & 0.010 & 7.774 & 0.010 & 3.135 & 0.033 & 3.159 & 0.034 \\
\hline 1356 & 15.228 & 0.003 & 15.418 & 0.003 & 7.655 & 0.001 & 7.735 & 0.001 & 3.082 & 0.036 & 3.113 & 0.036 \\
\hline
\end{tabular}

Table 12 PACS photometery of $\alpha$ Cet

\begin{tabular}{|c|c|c|c|c|c|c|c|c|c|c|c|c|}
\hline \multirow[t]{3}{*}{ OD } & \multicolumn{12}{|c|}{ filter } \\
\hline & \multicolumn{4}{|c|}{ blue $(70 \mu \mathrm{m})$} & \multicolumn{4}{|c|}{ green $(100 \mu \mathrm{m})$} & \multicolumn{4}{|c|}{ red $(160 \mu \mathrm{m})$} \\
\hline & \multicolumn{2}{|c|}{ with EVC } & \multicolumn{2}{|c|}{ without EVC } & \multicolumn{2}{|c|}{ with EVC } & \multicolumn{2}{|c|}{ without EVC } & \multicolumn{2}{|c|}{ with EVC } & \multicolumn{2}{|c|}{ without EVC } \\
\hline & flux[Jy] & error[Jy] & flux[Jy] & error[Jy] & flux[Jy] & error[Jy] & flux[Jy] & error[Jy] & flux[Jy] & error[Jy] & flux[Jy] & error[Jy] \\
\hline 259 & 5.071 & 0.002 & 5.104 & 0.002 & 2.498 & 0.006 & 2.514 & 0.006 & 1.009 & 0.009 & 1.015 & 0.009 \\
\hline 457 & 5.055 & 0.005 & 5.094 & 0.005 & 2.503 & 0.003 & 2.522 & 0.003 & 1.006 & 0.004 & 1.013 & 0.004 \\
\hline 614 & 5.036 & 0.006 & 5.058 & 0.006 & 2.515 & 0.003 & 2.525 & 0.003 & 1.004 & 0.040 & 1.008 & 0.040 \\
\hline 806 & 5.036 & 0.005 & 5.060 & 0.005 & 2.509 & 0.006 & 2.521 & 0.006 & 1.012 & 0.016 & 1.016 & 0.016 \\
\hline 1000 & 5.011 & 0.004 & 5.024 & 0.004 & 2.496 & 0.004 & 2.502 & 0.004 & 1.041 & 0.027 & 1.043 & 0.027 \\
\hline 1170 & 5.027 & 0.007 & 5.031 & 0.007 & 2.523 & 0.003 & 2.525 & 0.003 & 1.024 & 0.014 & 1.025 & 0.014 \\
\hline 1377 & 4.967 & 0.005 & 5.012 & 0.005 & 2.482 & 0.004 & 2.504 & 0.004 & 1.034 & 0.024 & 1.042 & 0.025 \\
\hline
\end{tabular}


Table 13 PACS photometery of $\beta$ And

\begin{tabular}{|c|c|c|c|c|c|c|c|c|c|c|c|c|}
\hline OD & \multicolumn{12}{|c|}{ filter } \\
\hline & \multicolumn{4}{|c|}{ blue $(70 \mu \mathrm{m})$} & \multicolumn{4}{|c|}{ green $(100 \mu \mathrm{m})$} & \multicolumn{4}{|c|}{$\operatorname{red}(160 \mu \mathrm{m})$} \\
\hline & \multicolumn{2}{|c|}{ with EVC } & \multicolumn{2}{|c|}{ without EVC } & \multicolumn{2}{|c|}{ with EVC } & \multicolumn{2}{|c|}{ without EVC } & \multicolumn{2}{|c|}{ with EVC } & \multicolumn{2}{|c|}{ without EVC } \\
\hline & flux [Jy] & error[Jy] & flux[Jy] & error[Jy] & flux [Jy] & error[Jy] & flux [Jy] & error[Jy] & flux[Jy] & error[Jy] & flux [Jy] & error[Jy] \\
\hline 414 & 5.842 & 0.007 & 5.872 & 0.007 & 2.891 & 0.005 & 2.906 & 0.005 & 1.139 & 0.024 & 1.144 & 0.024 \\
\hline 607 & 5.741 & 0.006 & 5.792 & 0.006 & 2.849 & 0.004 & 2.873 & 0.004 & 1.125 & 0.017 & 1.134 & 0.016 \\
\hline 777 & 5.802 & 0.009 & 5.853 & 0.009 & 2.875 & 0.004 & 2.899 & 0.004 & 1.121 & 0.022 & 1.129 & 0.022 \\
\hline 973 & 5.792 & 0.005 & 5.788 & 0.005 & 2.903 & 0.006 & 2.901 & 0.006 & 1.148 & 0.026 & 1.147 & 0.025 \\
\hline 1157 & 5.776 & 0.005 & 5.805 & 0.005 & 2.870 & 0.007 & 2.884 & 0.008 & 1.134 & 0.016 & 1.139 & 0.016 \\
\hline 1337 & 5.734 & 0.007 & 5.763 & 0.008 & 2.868 & 0.004 & 2.882 & 0.004 & 1.117 & 0.013 & 1.122 & 0.013 \\
\hline
\end{tabular}


Table 14 PACS photometery of $\gamma$ Dra

\begin{tabular}{|c|c|c|c|c|c|c|c|c|c|c|c|c|}
\hline \multirow[t]{3}{*}{ OD } & \multicolumn{12}{|c|}{ filter } \\
\hline & \multicolumn{4}{|c|}{ blue $(70 \mu \mathrm{m})$} & \multicolumn{4}{|c|}{ green $(100 \mu \mathrm{m})$} & \multicolumn{4}{|c|}{ red $(160 \mu \mathrm{m})$} \\
\hline & \multicolumn{2}{|c|}{ with EVC } & \multicolumn{2}{|c|}{ without EVC } & \multicolumn{2}{|c|}{ with EVC } & \multicolumn{2}{|c|}{ without EVC } & \multicolumn{2}{|c|}{ with EVC } & \multicolumn{2}{|c|}{ without EVC } \\
\hline & flux[Jy] & error[Jy] & flux [Jy] & error[Jy] & flux[Jy] & error[Jy] & flux[Jy] & error[Jy] & flux[Jy] & error[Jy] & flux [Jy] & error $[\mathrm{Jy}]$ \\
\hline 108 & 3.313 & 0.012 & 3.331 & 0.012 & 1.606 & 0.008 & 1.615 & 0.008 & 0.694 & 0.018 & 0.697 & 0.018 \\
\hline 191 & & & & & 1.659 & 0.008 & 1.667 & 0.008 & 0.722 & 0.018 & 0.725 & 0.019 \\
\hline 191 & & & & & 1.662 & 0.006 & 1.671 & 0.006 & 0.678 & 0.011 & 0.681 & 0.011 \\
\hline 191 & & & & & 1.639 & 0.007 & 1.647 & 0.007 & 0.692 & 0.014 & 0.695 & 0.015 \\
\hline 191 & & & & & 1.663 & 0.004 & 1.672 & 0.004 & 0.675 & 0.016 & 0.678 & 0.017 \\
\hline 191 & & & & & 1.649 & 0.005 & 1.658 & 0.005 & 0.670 & 0.006 & 0.673 & 0.006 \\
\hline 213 & 3.302 & 0.006 & 3.315 & 0.006 & & & & & 0.675 & 0.019 & 0.675 & 0.019 \\
\hline 244 & 3.318 & 0.011 & 3.333 & 0.011 & & & & & 0.649 & 0.008 & 0.651 & 0.008 \\
\hline 286 & 3.281 & 0.004 & 3.307 & 0.004 & & & & & 0.679 & 0.018 & 0.683 & 0.018 \\
\hline 300 & 3.294 & 0.004 & 3.313 & 0.004 & 1.629 & 0.003 & 1.639 & 0.003 & 0.669 & 0.010 & 0.673 & 0.010 \\
\hline 316 & 3.274 & 0.002 & 3.304 & 0.002 & & & & & 0.666 & 0.017 & 0.671 & 0.017 \\
\hline 345 & 3.298 & 0.002 & 3.323 & 0.002 & & & & & 0.688 & 0.033 & 0.693 & 0.033 \\
\hline 371 & 3.303 & 0.003 & 3.294 & 0.003 & & & & & 0.679 & 0.015 & 0.677 & 0.015 \\
\hline 400 & 3.307 & 0.008 & 3.323 & 0.008 & & & & & 0.697 & 0.020 & 0.700 & 0.021 \\
\hline 413 & 3.336 & 0.007 & 3.331 & 0.007 & & & & & 0.670 & 0.024 & 0.670 & 0.024 \\
\hline 413 & 3.336 & 0.006 & 3.345 & 0.006 & & & & & 0.690 & 0.015 & 0.691 & 0.015 \\
\hline 413 & 3.308 & 0.002 & 3.323 & 0.002 & & & & & 0.651 & 0.016 & 0.654 & 0.016 \\
\hline 414 & 3.323 & 0.005 & 3.339 & 0.005 & & & & & 0.636 & 0.008 & 0.638 & 0.008 \\
\hline 414 & 3.301 & 0.003 & 3.320 & 0.003 & & & & & 0.701 & 0.028 & 0.705 & 0.028 \\
\hline 414 & 3.307 & 0.006 & 3.329 & 0.006 & & & & & 0.664 & 0.008 & 0.668 & 0.008 \\
\hline 415 & 3.310 & 0.005 & 3.333 & 0.005 & & & & & 0.679 & 0.010 & 0.683 & 0.010 \\
\hline 415 & 3.295 & 0.003 & 3.324 & 0.003 & & & & & 0.668 & 0.016 & 0.673 & 0.016 \\
\hline 456 & 3.321 & 0.005 & 3.337 & 0.006 & & & & & 0.650 & 0.025 & 0.653 & 0.025 \\
\hline 483 & 3.327 & 0.003 & 3.329 & 0.003 & & & & & 0.685 & 0.016 & 0.685 & 0.016 \\
\hline 511 & 3.325 & 0.005 & 3.323 & 0.005 & & & & & 0.718 & 0.019 & 0.718 & 0.019 \\
\hline 539 & 3.329 & 0.009 & 3.322 & 0.009 & & & & & 0.653 & 0.012 & 0.651 & 0.012 \\
\hline 566 & 3.248 & 0.005 & 3.277 & 0.005 & 1.630 & 0.004 & 1.645 & 0.004 & 0.655 & 0.014 & 0.660 & 0.014 \\
\hline 607 & 3.283 & 0.004 & 3.310 & 0.004 & & & & & 0.677 & 0.014 & 0.682 & 0.014 \\
\hline 628 & 3.283 & 0.004 & 3.303 & 0.004 & & & & & 0.654 & 0.012 & 0.658 & 0.012 \\
\hline 662 & 3.273 & 0.007 & 3.290 & 0.007 & & & & & 0.679 & 0.018 & 0.682 & 0.019 \\
\hline 684 & 3.295 & 0.003 & 3.311 & 0.003 & & & & & 0.670 & 0.012 & 0.673 & 0.012 \\
\hline 715 & 3.279 & 0.002 & 3.298 & 0.002 & & & & & 0.674 & 0.021 & 0.677 & 0.021 \\
\hline 744 & 3.302 & 0.007 & 3.322 & 0.007 & & & & & 0.673 & 0.027 & 0.677 & 0.027 \\
\hline 764 & 3.220 & 0.006 & 3.329 & 0.007 & & & & & 0.669 & 0.007 & 0.689 & 0.007 \\
\hline 792 & 3.200 & 0.002 & 3.320 & 0.002 & & & & & 0.618 & 0.020 & 0.638 & 0.020 \\
\hline 826 & 3.285 & 0.004 & 3.311 & 0.004 & & & & & 0.670 & 0.020 & 0.674 & 0.021 \\
\hline 849 & 3.275 & 0.003 & 3.302 & 0.003 & 1.621 & 0.008 & 1.634 & 0.008 & 0.674 & 0.015 & 0.679 & 0.015 \\
\hline 887 & 3.298 & 0.006 & 3.292 & 0.006 & & & & & 0.704 & 0.011 & 0.703 & 0.011 \\
\hline 906 & 3.236 & 0.005 & 3.264 & 0.005 & & & & & 0.663 & 0.034 & 0.668 & 0.035 \\
\hline 936 & 3.269 & 0.006 & 3.292 & 0.006 & & & & & 0.672 & 0.015 & 0.675 & 0.016 \\
\hline 967 & 3.261 & 0.006 & 3.269 & 0.006 & & & & & 0.655 & 0.014 & 0.657 & 0.014 \\
\hline 1000 & 3.283 & 0.005 & 3.288 & 0.005 & & & & & 0.712 & 0.019 & 0.713 & 0.019 \\
\hline 1028 & 3.268 & 0.008 & 3.297 & 0.008 & & & & & 0.674 & 0.013 & 0.679 & 0.013 \\
\hline 1049 & 3.302 & 0.006 & 3.305 & 0.006 & & & & & 0.712 & 0.011 & 0.713 & 0.011 \\
\hline 1076 & 3.227 & 0.005 & 3.281 & 0.005 & & & & & 0.677 & 0.016 & 0.685 & 0.016 \\
\hline 1109 & 3.283 & 0.006 & 3.305 & 0.006 & & & & & 0.651 & 0.017 & 0.655 & 0.017 \\
\hline 1137 & 3.280 & 0.007 & 3.281 & 0.007 & 1.632 & 0.004 & 1.633 & 0.004 & 0.684 & 0.021 & 0.684 & 0.021 \\
\hline 1157 & 3.264 & 0.005 & 3.280 & 0.005 & & & & & 0.683 & 0.026 & 0.686 & 0.026 \\
\hline 1184 & 3.279 & 0.004 & 3.308 & 0.004 & & & & & 0.669 & 0.013 & 0.674 & 0.013 \\
\hline 1216 & 3.254 & 0.003 & 3.283 & 0.003 & & & & & 0.667 & 0.029 & 0.672 & 0.029 \\
\hline 1244 & 3.269 & 0.005 & 3.280 & 0.005 & & & & & 0.676 & 0.008 & 0.678 & 0.008 \\
\hline 1275 & 3.237 & 0.004 & 3.265 & 0.004 & & & & & 0.667 & 0.013 & 0.672 & 0.013 \\
\hline 1308 & 3.127 & 0.008 & 3.121 & 0.007 & & & & & 0.664 & 0.024 & 0.663 & 0.024 \\
\hline 1334 & 3.244 & 0.004 & 3.275 & 0.004 & 1.622 & 0.004 & 1.639 & 0.004 & 0.664 & 0.010 & 0.669 & 0.010 \\
\hline 1355 & 3.257 & 0.005 & 3.276 & 0.005 & & & & & 0.652 & 0.012 & 0.655 & 0.012 \\
\hline 1399 & 3.253 & 0.004 & 3.269 & 0.004 & & & & & 0.674 & 0.042 & 0.676 & 0.042 \\
\hline 1418 & 3.286 & 0.003 & 3.288 & 0.003 & & & & & 0.692 & 0.019 & 0.692 & 0.019 \\
\hline 1427 & 3.239 & 0.008 & 3.262 & 0.008 & & & & & 0.686 & 0.021 & 0.690 & 0.021 \\
\hline 1444 & 3.249 & 0.002 & 3.271 & 0.002 & & & & & 0.665 & 0.025 & 0.669 & 0.025 \\
\hline
\end{tabular}

